"This document is the Accepted Manuscript version of a Published Work that appeared in final form in Macromolecules 2016, 49, 6285-6295, copyright (C) American Chemical Society after peer review and technical editing by the publisher. To access the final edited and published work see DOI: 10.1021/acs.macromol.6b01449. This article may be used for noncommercial purposes in accordance with the ACS guidelines published at http://pubs.acs.org/page/policy/articlesonrequest/index.html]." 


\title{
Terpolymers derived from Limonene Oxide and Carbon Dioxide: Access to Cross-Linked Polycarbonates with Improved Thermal Properties
}

\author{
Carmen Martín ${ }^{\dagger}$ and Arjan W. Kleij ${ }^{*,+, *}$
}

$\dagger$ Institute of Chemical Research of Catalonia (ICIQ), the Barcelona Institute of Science and Technology, Av. Països Catalans 16, 43007 - Tarragona, Spain.

Catalan Institute of Research and Advanced Studies (ICREA), Pg. Lluís Companys 23, 08010 Barcelona, Spain.

Table of contents entry:

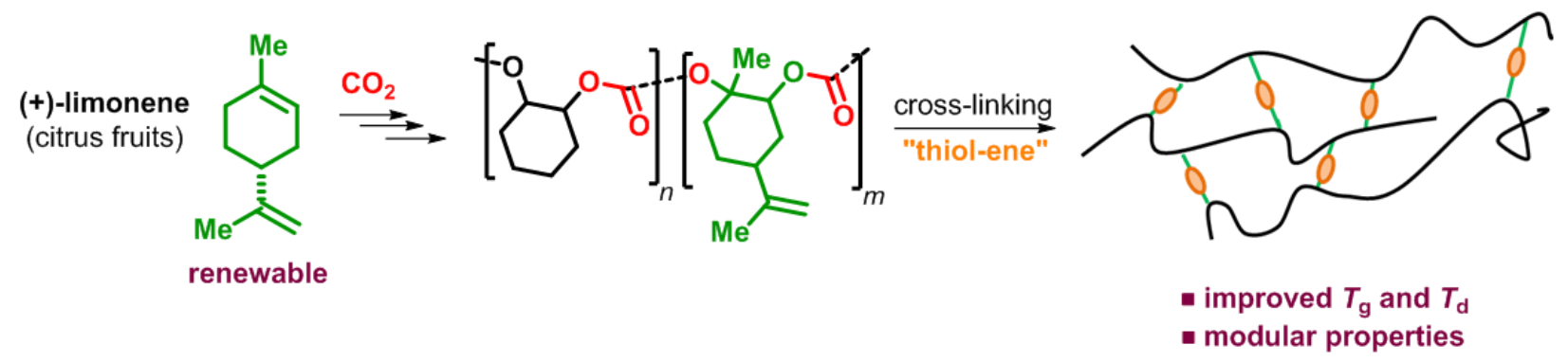


ABSTRACT: The formation of bio-derived materials is gaining momentum in academic and industrial research as a consequence of depleting petroleum resources, providing mid- and longterm alternative sustainable materials. In this context, we have prepared a series of terpolymers derived from the renewable terpene limonene producing polycarbonates with a controlled and variable ratio of incorporated nonfunctional cyclohexene oxide $(\mathrm{CHO})$ and functional limonene oxide (LO) monomers. As catalyst, a simple binary combination of an Al(III) aminotriphenolate complex and PPNCl (PPN = bis(triphenyl)phosphine-ammonium) has been used to afford the targeted, partially bio-based polycarbonates with typically high carbonate content $(>95 \%)$, good conversions and a controllable amount of olefinic groups in the terpolymer backbone. Crosslinked polymers (CLP) have been easily obtained from these terpolymers through thiol-ene click reactions allowing for the preparation of interconnected networks with improved thermal properties with their $T_{\mathrm{d}}$ in the range of $250-280^{\circ} \mathrm{C}$ and with glass transitions $\left(T_{\mathrm{g}}\right)$ of up to $150^{\circ} \mathrm{C}$. A detailed analysis of the cross-linked polycarbonates demonstrates a clear relation between the percentage of potential cross-linking groups in the terpolymer precursors and the physicochemical properties including solubility, rigidity and thermal stability. 


\section{INTRODUCTION}

Polymers are ubiquitous building blocks for a range of consumer-based products providing the necessary plastics, coatings and fibers that increase the quality of life. Therefore, the development of new (catalytic) strategies to obtain synthetic polymers with new and improved properties continues to attract huge interest. The conversion of natural resources into valueadded polymers ${ }^{1}$ is gaining momentum due to environmental issues, fore-casted depleting fossil resources and more strict waste legislation. The use of carbon dioxide $\left(\mathrm{CO}_{2}\right)$ as a carbon feed stock in organic synthesis ${ }^{2}$ and the replacement of fossil fuel feed stocks for natural, renewable ones $^{3}$ in polymer synthesis have become mature areas of research. In the latter context, polycarbonates produced by ring-opening copolymerization of epoxides and $\mathrm{CO}_{2}$ represent promising a type of materials. ${ }^{4}$ For instance, epoxide/ $\mathrm{CO}_{2}$ coupling processes have led to the synthesis of new polymers through the use of bio-based monomers based on terpenes ${ }^{5}$ or fatty acids. ${ }^{6}$ These new approaches enable the synthesis of polymers with diverse, new architectures and properties potentially useful in applications that go beyond bench-scale. Thus, beside the conventional alternating copolymerization of epoxides and $\mathrm{CO}_{2}$ (copolymers, Figure 1a), novel polymers have been synthesized by sequential epoxide addition $\left(\mathrm{CO}_{2}\right.$-based block copolymers, Figure $1 b)^{7,8}$ or by random incorporation of multiple epoxide monomers (terpolymers; Figure 1c). ${ }^{9}, 10,11$ Interestingly, these block copolymers and terpolymers have been prepared with the idea

to use the pendant functional groups for post-polymerization modifications. A wide range of such functional (ter)polymer examples exist incorporating different polymer side chain groups such as alcohols, carboxylic acids, amines, ionic fragments and alkenes. The latter category has been popular in this respect as it allows for easy conversion by thiol-ene "click" chemistry" to modify the polymer properties. ${ }^{8,10,12}$ 
(a) co-polymer

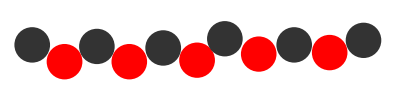

(b) block co-polymer

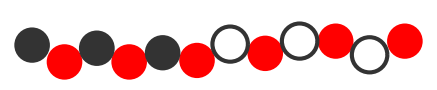

(c) terpolymer

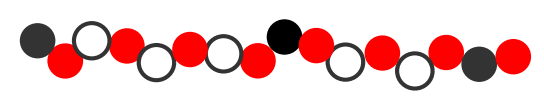

Figure 1. Structures of different $\mathrm{CO}_{2}$-based polycarbonates: (a) Co-polymers, (b) Block copolymers and (c) Terpolymers. The red circles represent the carbonate units, whereas the black and white circles are different incorporated epoxide monomers.

The easy click chemistry and other post-polymerization reactions can be used to prepare new functionalized polymers but also offer opportunities towards the formation of cross-linked polymers by interconnecting different polymer chains (intermolecular process) or fragments (intramolecular process). Such cross-linking may give polymers with improved mechanical strength and thermal stability, as well as polymer nanoparticles with many applications including data storage technology. ${ }^{13,14}$ However, examples of cross-linked polycarbonates based on functional monomers remain rather limited to date. An interesting example was reported by the Coates group, describing the efficient transformation of a linear polycarbonate with pendent alkene groups into organic nanoparticles through well-established olefin cross-metathesis reactions (Figure 2a). ${ }^{9}$ The group of Darensbourg utilized a radical initiated thiol-ene approach to prepare cross-linked materials using a rigid pentaerythriol tetrakis(mercaptoacetate) (Figure 2b). ${ }^{15}$ Another recent example of this thiol-ene chemistry is the formation of a rigidified polymer reported by Pescarmona et al. that exhibited a high glass transition temperature $\left(T_{\mathrm{g}}=130^{\circ} \mathrm{C}\right.$, Figure 2c). ${ }^{16}$ In this latter example a flexible dithiol linker was employed to connect the olefin fragments present in the oligomeric carbonates. All these cross-linked polymers are based on 
petroleum feed stocks, and thus the development of new cross-linked materials derived from natural resources remains an interesting target to provide more sustainable alternatives.

(a)

Coates (2007)

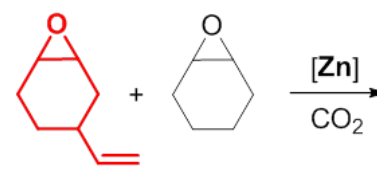

Darensbourg (2015)

(b)

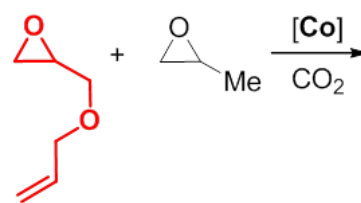

Pescarmona (2015)

(c)

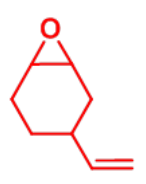

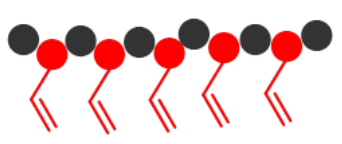

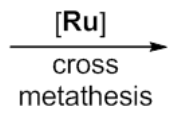

metathesis
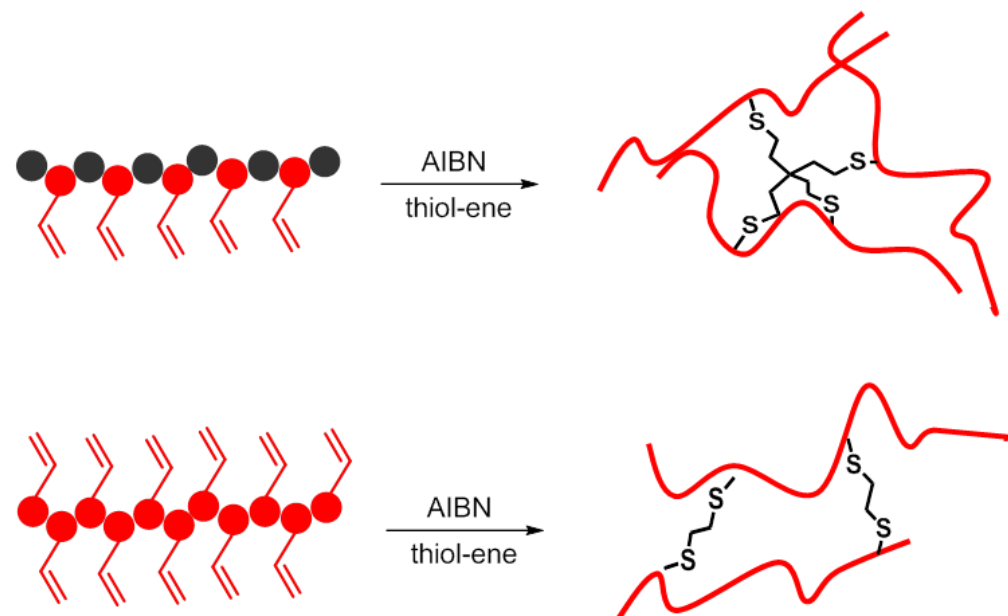
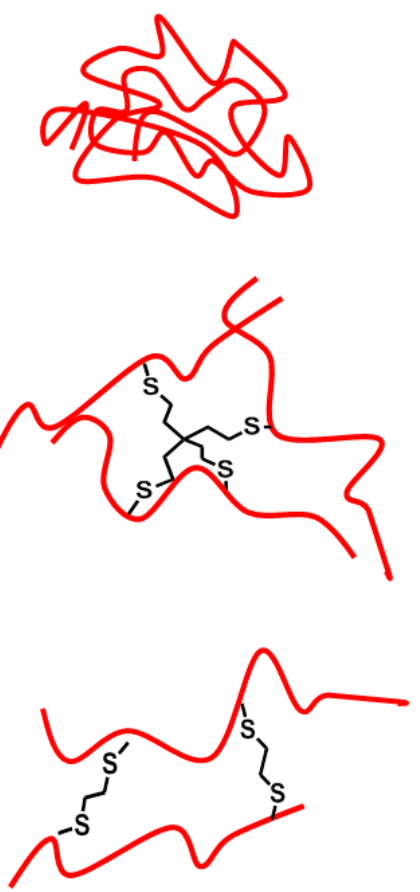

This Work:

(d)<smiles>C=C(C)C1CCC2(C)OC2C[CH+]C1C</smiles>

limonene oxide renewable \& functional

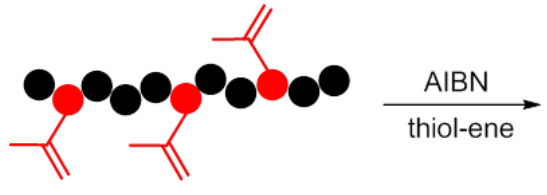

functional terpolymers

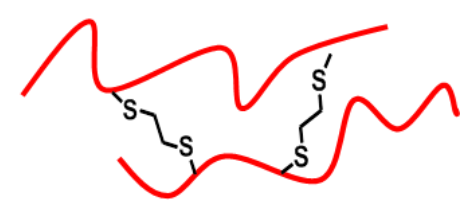

Figure 2. Schematic drawings of cross-linked polymers derived from petroleum sources (a-c) and a functional terpolymer derived from the naturally occuring limonene (d). Note that here the black and red units represent a full repeat unit based on an epoxide monomer and $\mathrm{CO}_{2}$. 
As mentioned before, there are only few examples of $100 \%$ bio-based polycarbonates known in the literature where natural oils have been used as co-monomers. ${ }^{5,6}$ In this regard, the first copolymerization reaction using terpene-derived epoxides (i.e., limonene oxide, LO) and $\mathrm{CO}_{2}$ was described by Coates and co-workers utilizing highly active $\beta$-diiminate (BDI) zinc catalysts, and they succeeded in the stereoselective conversion of trans-LO. ${ }^{5 a}$ Furthermore, we recently communicated the use of $\mathrm{Al}($ aminotrisphenolate)/PPNCl $[\mathrm{PPN}=$ bis(triphenylphosphine)iminium] binary catalysts for stereoregular $\mathrm{LO} / \mathrm{CO}_{2}$ copolymerization. ${ }^{17}$ As far as we know, the synthesis of $\mathrm{CO}_{2}$-based terpolymers derived from renewable terpenes is still an unexplored area. Inspired by our previous results in LO copolymerization, we set out to extend this approach towards the preparation of (partially) renewable terpolymers. Here we disclose an efficient catalytic method for the synthesis of new terpolymers based on cyclohexene oxide $(\mathrm{CHO}), \mathrm{LO}$ and $\mathrm{CO}_{2}$ with controllable incorporation of both functional (LO) and nonfunctional (CHO) monomers in the polymer backbone. The olefin groups in the resultant terpolymers allow for post-polymerization cross-linking by thiol-ene click chemistry, and our modular strategy provides networks derived from terpenes with high thermal stability and rigidity, and offers alternatives for polycarbonate based materials.

\section{EXPERIMENTAL SECTION}

General Considerations. All water sensitive operations were carried out under a nitrogen atmosphere using an MBraun glovebox, standard vacuum-line and Schlenk techniques. Solvents were purchased from Sigma-Aldrich as HPLC grade and dried by means of an MBraun MBSPS800 purification system. All reagents were purchased from commercial suppliers (Aldrich and Acros) and used as received unless stated otherwise. FT-IR measurements were 
performed on a Bruker Optics FTIR Alpha spectrometer equipped with a DTGS detector, $\mathrm{KBr}$ beam-splitter at $4 \mathrm{~cm}^{-1}$ resolution. Matrix-assisted laser desorption/ionization time-of-flight mass spectrometry (MALDI-TOF-MS) was performed by the Research Support Group at ICIQ on a BRUKER Autoflex spectrometer (more details are provided in the Supporting Information). NMR spectra were recorded on a Bruker AV-400 spectrometer and referenced to the residual NMR solvent signals. Glass transition temperatures $\left(T_{\mathrm{g}}\right)$ were measured under $\mathrm{N}_{2}$ atmosphere using a Mettler Toledo equipped, model DSC822e. Samples were weighed into $40 \mu \mathrm{L}$ aluminum crucibles and subjected to three heating cycles covered the range from $20^{\circ} \mathrm{C}$ to $150-190^{\circ} \mathrm{C}$ at a heating rate of $10^{\circ} \mathrm{C} / \mathrm{min}$. Thermo-gravimetric analyses were recorded under a $\mathrm{N}_{2}$ atmosphere using a Mettler Toledo equipped; model TGA/SDTA851 with a heating rate of $10^{\circ} \mathrm{C} / \mathrm{min}$. Molecular weights $\left(M_{\mathrm{n}}\right)$ and their distributions $(\nexists)$ of the terpolymers were determined by gel permeation chromatography (GPC) at the Instituto de Ciencia y Tecnología de Polímeros (CSIC), Madrid. GPC analyses were carried out with Styragel $(300 \times 7.8 \mathrm{~mm}, 5 \mu \mathrm{m}$ nominal particle size) water columns. Measurements were performed at $35^{\circ} \mathrm{C}$ at a flow rate of $1 \mathrm{~mL} / \mathrm{min}$ using a RI detector (Waters, model 410) and using THF as solvent. Samples were analyzed at a concentration of $4 \mathrm{mg} \cdot \mathrm{mL}^{-1}$ after filtration through a $0.22 \mu \mathrm{m}$ pore-size. $M_{\mathrm{n}}, M_{\mathrm{w}}$ and $M_{\mathrm{w}} / M_{\mathrm{n}}(\nexists)$ data were derived from the RI signal by a calibration curve based on polystyrene standards.

Reagents and Catalyst. Commercial $\mathrm{CO}_{2}$ was obtained from Praxair and used without further purification. The commercially available epoxide substrates 1,2-epoxy-cyclohexane (CHO) and $(R)$-limone oxide $(\mathrm{LO})$ were used after distillation over calcium hydride, $\mathrm{CaH}_{2}$. Cis-limonene ${ }^{18}$ oxide was obtained following a literature procedure and purified by distillation over $\mathrm{CaH}_{2}$. Commercially available nucleophilic additives bis(triphenylphosphine)iminium chloride $(\mathrm{PPNCl})$ was purified by recrystallization from dichloromethane and dried in vacuo at $40^{\circ} \mathrm{C}$ for 
24 h. Tris(3,5-dimethyl-2-hydroxybenzyl)amine and its aluminium complex $\left(\mathbf{A l} \mathbf{l}^{\mathbf{M e}}\right)$ were prepared as described previously ${ }^{19}$ and used after drying under vacuum at $40^{\circ} \mathrm{C}$ for $24 \mathrm{~h}$.

General Catalytic Procedure for the Copolymerization of $\mathrm{CO}_{2}$ and Epoxides. All reactions were prepared in a glovebox using a $30 \mathrm{~mL}$ stainless-steel Berghof reactor. In a typical experiment, the catalyst $(0.05 \mathrm{mmol})$, co-catalyst/nucleophile $(0.025 \mathrm{mmol})$ and $10 \mathrm{mmol}$ of epoxide ( $0.98 \mathrm{~g}$ of $\mathrm{CHO}$ or $1.52 \mathrm{~g}$ of $\mathrm{LO})$ were added into the teflon reaction vessel. Three cycles of pressurization and depressurization of the reactor ( 5 bar) were carried out before finally stabilizing the pressure at 15 bar. The reaction was stirred at $45^{\circ} \mathrm{C}\left(40^{\circ} \mathrm{C}\right.$ inside the reactor) for 48 h. Finally, the reaction was stopped and the autoclave was allowed to cool down to room temperature before venting. The crude reaction mixture was analyzed by ${ }^{1} \mathrm{H}$ NMR spectroscopy to determine the conversion to polymer and the amount of ether linkages.

General Catalytic Procedure for the CHO-LO- $\mathrm{CO}_{2}$ Terpolymerization Reactions. The experiments were performed as mentioned above for the copolymerization processes using 0.05 mmol of catalyst, $0.025 \mathrm{mmol}$ of co-catalyst and $10 \mathrm{mmol}$ of the corresponding mixture of LO and CHO. For example, the terpolymer containing 30\% of the LO monomer incorporated was prepared from a mixture of $5 \mathrm{mmol}$ of $\mathrm{CHO}(0.49 \mathrm{~g})$ and $5 \mathrm{mmol}$ of $\mathrm{LO}(0.76 \mathrm{~g})$ at $15 \mathrm{bar}, 45^{\circ} \mathrm{C}$ $\left(40{ }^{\circ} \mathrm{C}\right.$ inside the reactor) during $48 \mathrm{~h}$.

Kinetic Experiments. All reactions for the polymerization control experiments and kinetic studies were prepared in the glovebox using a $30 \mathrm{~mL}$ stainless-steel Berghof reactor. The teflon reaction vessel was charged with $0.50 \mathrm{~mol} \%$ of $\mathbf{A l}^{\mathrm{Me}}$ catalyst, $0.25 \mathrm{~mol} \%$ of co-catalyst (PPNCl) and $3.5 \mathrm{mmol}$ of the epoxide mixture (1:1 ratio; $0.17 \mathrm{~g}$ of $\mathrm{CHO}$ and $0.27 \mathrm{~g}$ of $\mathrm{LO}$ ). Three cycles of pressurization and depressurization of the reactor ( 5 bar) were carried out before finally stabilizing the pressure at 15 bar. Parallel reactions were stirred at $45^{\circ} \mathrm{C}\left(40^{\circ} \mathrm{C}\right.$ inside the 
reactor) for different reaction times (from 2 to $48 \mathrm{~h}$ ). For each reaction time, stirring was stopped and the autoclave was cooled down to room temperature before venting. The conversion of the substrate was examined by ${ }^{1} \mathrm{H}$ NMR spectroscopy $\left(\mathrm{CDCl}_{3}\right)$ of an aliquot taken from the crude reaction mixture and using as internal standard 1,4-dimethoxybenzene (typically 5.0 mol \% with respect to the epoxide substrate).

Isolation of the Polymers. The crude reaction mixture was extracted with dichloromethane and the solution allowed to evaporate. The crude mixture was then re-dissolved in a minimal amount of dichloromethane and the polymer precipitated with a $1 \mathrm{M}$ solution of hydrochloric acid in methanol. Upon settling, the supernatant solution was decanted and discarded. Then the polymer was washed with a solution of methanol. Finally the purified polymer was dried in vacuo at $40{ }^{\circ} \mathrm{C}$ overnight and analyzed by ${ }^{1} \mathrm{H}$ and ${ }^{13} \mathrm{C}\left\{{ }^{1} \mathrm{H}\right\}$ NMR, IR, DSC, TGA and GPC. The MALDI-TOF assignment for various terpolymers was done taking into account the relative ratios of $\mathrm{CHO} / \mathrm{LO}$ monomers incorporated as determined by ${ }^{1} \mathrm{H}$ NMR spectroscopy.

\section{General Procedure for the Cross-Linking Process through Thiol-Ene Click Chemistry.} All reactions were carried out under inert conditions using standard vacuum-line and Schlenk techniques. A radical initiator (0.20 equiv. of AIBN; 2,2'-azobis(2-methylpropionitrile) $v s$ olefins units in polymers) and $150 \mathrm{mg}$ of the corresponding polymer were transferred into a Schlenk tube equipped with a Teflon stirring bar. Then, the solvent (tetrahydrofuran, $1.0 \mathrm{~mL}$ ) and 1,2-ethanedithiol ( 0.50 equiv. vs olefin units in the polymers) were added. ${ }^{20}$ The reaction mixture was heated in an oil bath at $70^{\circ} \mathrm{C}$ and after $12 \mathrm{~h}$ the volatiles were removed in vacuo. Afterward, the crude reaction mixture was washed three times with methanol. Finally, the purified polymer was dried in vacuo and analyzed by ${ }^{1} \mathrm{H}$ and ${ }^{13} \mathrm{C}\left\{{ }^{1} \mathrm{H}\right\} \mathrm{NMR}, \mathrm{IR}, \mathrm{GPC}, \mathrm{DSC}$, TGA. 


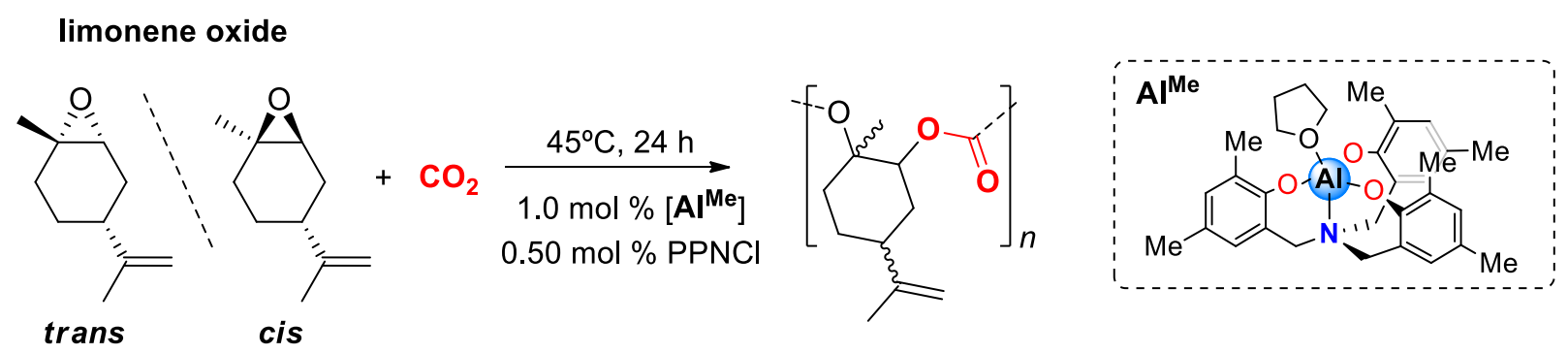

Figure 3. Previously developed $\mathrm{LO} / \mathrm{CO}_{2}$ copolymerization process using the binary catalyst

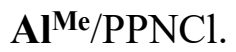

\section{RESULTS AND DISCUSSION}

Terpolymer Synthesis using the Binary Catalyst $\mathbf{A l}^{\mathrm{Me}} / \mathbf{P P N C l}$. Previously we reported the use of Al-based amino(triphenolate) complexes with PPNCl as binary catalysts for the coupling reaction between $\mathrm{CO}_{2}$ and limonene oxide (LO) (Figure 3). ${ }^{17}$ The aluminum complex with peripheral methyl substituents has proven to be an efficient catalyst for the synthesis of stereoregular LO-based polycarbonates with $M_{\mathrm{n}}$ 's up to around $10.6 \mathrm{~kg} / \mathrm{mol}(\nexists \sim 1.4)$ after $24 \mathrm{~h}$ at $45^{\circ} \mathrm{C}$ and 5 bar operating pressure under neat conditions using $1.0 \mathrm{~mol} \% \mathrm{of}^{\mathbf{M e}}$ and $0.50 \mathrm{~mol}$ $\%$ of PPNCl with respect to the substrate. Furthermore, the use of this catalytic system has shown kinetic differences between the conversions of the cis- and trans-LO monomer, with the latter one being more slowly consumed. Based on these observations, we decided to probe a similar approach towards terpolymers based on $\mathrm{CO}_{2}, \mathrm{CHO}$ and the renewable substrate cis-LO. Initially we assessed the potential terpolymerization process under similar conditions used for the $\mathrm{LO} / \mathrm{CO}_{2}$ copolymerization process, i.e. using $1.0 \mathrm{~mol} \%$ of $\mathbf{A l}^{\mathrm{Me}}$ and $0.50 \mathrm{~mol} \%$ of $\mathrm{PPNCl}$, and performing the reaction at $45^{\circ} \mathrm{C}$ and 10 bar pressure. Upon using a starting ratio of $1: 1$ between both epoxides, we clearly observed the formation of a polymer having both monomers 
incorporated. The conversion $(24 \mathrm{~h} ; 75 \%)$ of the substrate and the percentage of LO incorporated (30\%; by signal integration) could be calculated by ${ }^{1} \mathrm{H}$ NMR spectroscopic analysis of an aliquot taken from the reaction mixture (Supporting Information, Figure S23). Thermal analysis showed several glass transition temperatures for this $\mathrm{CO}_{2}$-based terpolymer $\left(T_{\mathrm{g}}=54,72\right.$ and $126^{\circ} \mathrm{C}$, respectively, Supporting Information Figure S57). The presence of different $T_{\mathrm{g}}$ values observed by differential scanning calorimetry analysis (DSC) may be a result of a poor control over the terpolymerization process leading to a rather heterogenous combination of terpolymeric species. With the aim to gain improved control over the coupling product between $\mathrm{CO}_{2}$ and both epoxides, we decided to use lower amounts of $\mathbf{A l}^{\mathrm{Me}}$ and PPNCl (0.50 and $0.25 \mathrm{~mol} \%$, respectively) as well as a slightly lower reaction temperature $\left(40^{\circ} \mathrm{C}\right)$ and higher initial pressure $\left(p\left(\mathrm{CO}_{2}\right)^{\mathrm{o}}=15 \mathrm{bar}\right)$. Under these latter conditions, the reaction seems to proceed more selectively though a longer reaction time was necessary to obtain appreciable monomer conversions. Importantly, the selective formation of a polycarbonate containing 30\% of incorporated $\mathrm{LO}$ and with only one $T_{\mathrm{g}}\left(95^{\circ} \mathrm{C}\right)$ was noted. However, the molecular weight that was observed was rather modest $M_{\mathrm{n}}=4.0 \mathrm{~kg} / \mathrm{mol}$ with a narrow polydispersity $(\nexists=1.48$; see Table 1 entry 4$)$. On the basis of this result, we used similar conditions to access various $\mathrm{CHO} / \mathrm{LO}$ based terpolymers with different percentages of LO incorporated (Table 1). Variation of the relative concentrations of both $\mathrm{CHO}$ and $\mathrm{LO}$ monomers in reactions led to different polycarbonates with percentages of incorporated $\mathrm{LO}$ monomer ranging from $10-40 \%$ (Table 1). Additionally, the $\mathrm{CHO} / \mathrm{CO}_{2}$ and $\mathrm{LO} / \mathrm{CO}_{2}$ copolymers were also prepared under the same conditions to compare their properties with the terpolymer ones. ${ }^{21}$ 
Table 1. Terpolymerization of $\mathrm{CHO}$, cis- $\mathrm{LO}$ and $\mathrm{CO}_{2}$ using $\mathbf{A l}^{\mathrm{Me}}$ as Catalyst and $\mathrm{PPNCl}$ as Nucleophilic Additive. ${ }^{a}$

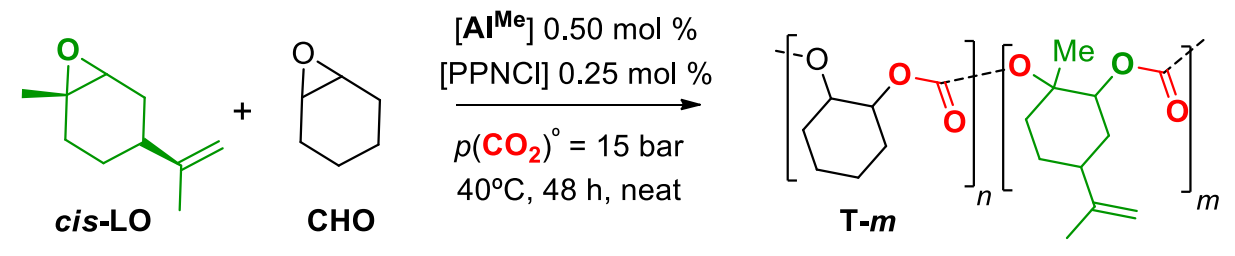

\begin{tabular}{|c|c|c|c|c|c|c|c|c|c|}
\hline Entry & Polymer & $\mathrm{CHO}: \mathbf{L O}^{b}$ & $\begin{array}{l}\text { Conv. } \\
(\%)^{c}\end{array}$ & $\begin{array}{c}\text { carbonate linkages } \\
\qquad(\%)^{d}\end{array}$ & $\begin{array}{l}n: m \\
(\%)^{d}\end{array}$ & $M_{\mathrm{n}}{ }^{e, f}$ & $\boldsymbol{D}^{e}$ & $\begin{array}{c}T_{\mathrm{g}} \\
\left({ }^{\circ} \mathbf{C}\right)^{g}\end{array}$ & $\begin{array}{c}T_{\mathbf{d}} \\
\left({ }^{\circ} \mathbf{C}\right)^{h}\end{array}$ \\
\hline 1 & $\mathbf{P}-\mathbf{0}^{i}$ & $1: 0$ & 77 & 99 & $100: 0$ & 11.9 & 1.49 & 114 & 249 \\
\hline 2 & T-10 & $2: 1$ & 70 & 99 & $90: 10$ & 8.22 & 1.24 & 111 & 263 \\
\hline 3 & T-20 & $1.5: 1$ & 68 & 98 & $83: 17$ & 5.82 & 1.31 & 109 & 248 \\
\hline 4 & T-30 & $1: 1$ & 75 & 95 & $69: 32$ & 3.96 & 1.48 & 96 & 210 \\
\hline 5 & T-40 & $1: 2$ & 73 & 95 & $56: 42$ & 3.58 & 1.34 & 83 & 228 \\
\hline 6 & $\mathbf{P}-100^{i}$ & $0: 1$ & 65 & 93 & $0: 100$ & 3.69 & 1.34 & 72 & 226 \\
\hline
\end{tabular}

${ }^{a}$ Reaction conditions: $\mathrm{CHO}$ and LO $\left(10 \mathrm{mmol}\right.$ in total, molar ratio indicated in the table), $\mathbf{A l}^{\mathrm{Me}} 0.50 \mathrm{~mol} \%, \mathrm{PPNCl} 0.25 \mathrm{~mol} \%, 40^{\circ} \mathrm{C}$, $p\left(\mathrm{CO}_{2}\right)^{\mathrm{o}}=15 \mathrm{bar}, 30 \mathrm{~mL}$ autoclave, neat conditions, $48 \mathrm{~h} .{ }^{b} \mathrm{CHO}$ and $\mathrm{LO}$ molar ratios used in the catalytic reaction. ${ }^{c}$ Determined by ${ }^{1} \mathrm{H}$ NMR analysis of the crude reaction mixture. ${ }^{d}$ Determined by ${ }^{1} \mathrm{H}$ NMR analysis of the isolated polymer. ${ }^{e}$ Determined by gel permeation chromatography (GPC) analysis in THF using polystyrene standards. ${ }^{f}$ In $\mathrm{kg} / \mathrm{mol}$. ${ }^{g}$ Determined by differential scanning calorimetry (DSC). ${ }^{h}$ Decomposition temperature $\left(T_{\mathrm{d}}\right)$ determinated by thermogravimetric analysis (TGA) at $10 \%$ weight loss of the polymer analyte. ${ }^{i} \mathrm{CHO} / \mathrm{CO}_{2}$ and $\mathrm{LO} / \mathrm{CO}_{2}$ copolymers (P-0 and $\mathbf{P - 1 0 0}$, respectively) were prepared under the same conditions. 
The data in Table 1 show that the percentage of LO incorporated in these terpolymers has a significant effect on the molecular weight $\left(M_{\mathrm{n}}\right)$ as well as on the glass transition temperature $\left(T_{\mathrm{g}}\right)$. At higher percentages of LO incorporated in the terpolymer products, lower molecular weights are found and concomitantly lower $T_{\mathrm{g}}$ values. These observations are to be expected when the amount of LO is increased in the initial reaction mixture as this monomer typically shows lower conversion kinetics compared to $\mathrm{CHO}$.

In order to gain more insight into the terpolymerization process, the $\mathrm{CO}_{2} / \mathrm{CHO} / \mathrm{LO}$ coupling process was followed in time. Such kinetic experiments are usually performed to assess whether diblock copolymers or terpolymeric structures are formed. ${ }^{22}$ This type of analysis is useful to get more detailed information about the reactivity of all involved monomers and the monomer composition in the chain as to discriminate between a homogeneous or a gradient type of terpolymer. ${ }^{15,23}$ We thus carried out a set of experiments with an equimolar CHO/LO mixture at $40^{\circ} \mathrm{C}$ and 15 bar and followed the terpolymerization process in time. Reactions were stopped at different time intervals and the reaction mixtures were analyzed by ${ }^{1} \mathrm{H}$ NMR spectroscopy to determine the consumption of each epoxide monomer (Supporting Information, Table S1). Figure 4 shows the conversion of both $\mathrm{CHO}$ and $\mathrm{LO}$ in this terpolymerization process. Clearly, $\mathrm{CHO}$ reacts much faster than $\mathrm{LO}$, delivering a polycarbonate with an incorporation ratio $\mathrm{CHO} / \mathrm{LO}$ of $74: 26$ determined by ${ }^{1} \mathrm{H}$ NMR signal integration. Despite the fact that $\mathrm{CHO}$ reacts significantly faster in this terpolymerization, LO incorporation into the polymer backbone occurs from the early stage ( $12 \%$ on average; after $2 \mathrm{~h}, 14 \%$ total monomer conversion) to the end of the process ( $26 \%$ on average; after $48 \mathrm{~h}$ with a $70 \%$ total monomer conversion). Therefore, the polymer seemingly has the functional monomer LO reasonably distributed throughout the entire chain. To further substantiate a random distribution of the LO monomer, the ratio between the 
net consumption of $\mathrm{CHO}$ and $\mathrm{LO}$ monomers was followed in time providing relative incorporation kinetics (Figure 5). This graph thus gives an impression of the relative rates of incorporation of both monomers, and the terpolymer that was produced has apparently a high $\mathrm{CHO} / \mathrm{LO}$ incorporation ratio (7:1) at the initial stage of the reaction (first $2 \mathrm{~h}$ period), which then quickly decays to lower $\mathrm{CHO} / \mathrm{LO}$ incorporation rates in subsequent one hour time intervals $(\mathrm{t}=$ $3,4,5$ and $6 \mathrm{~h})$.

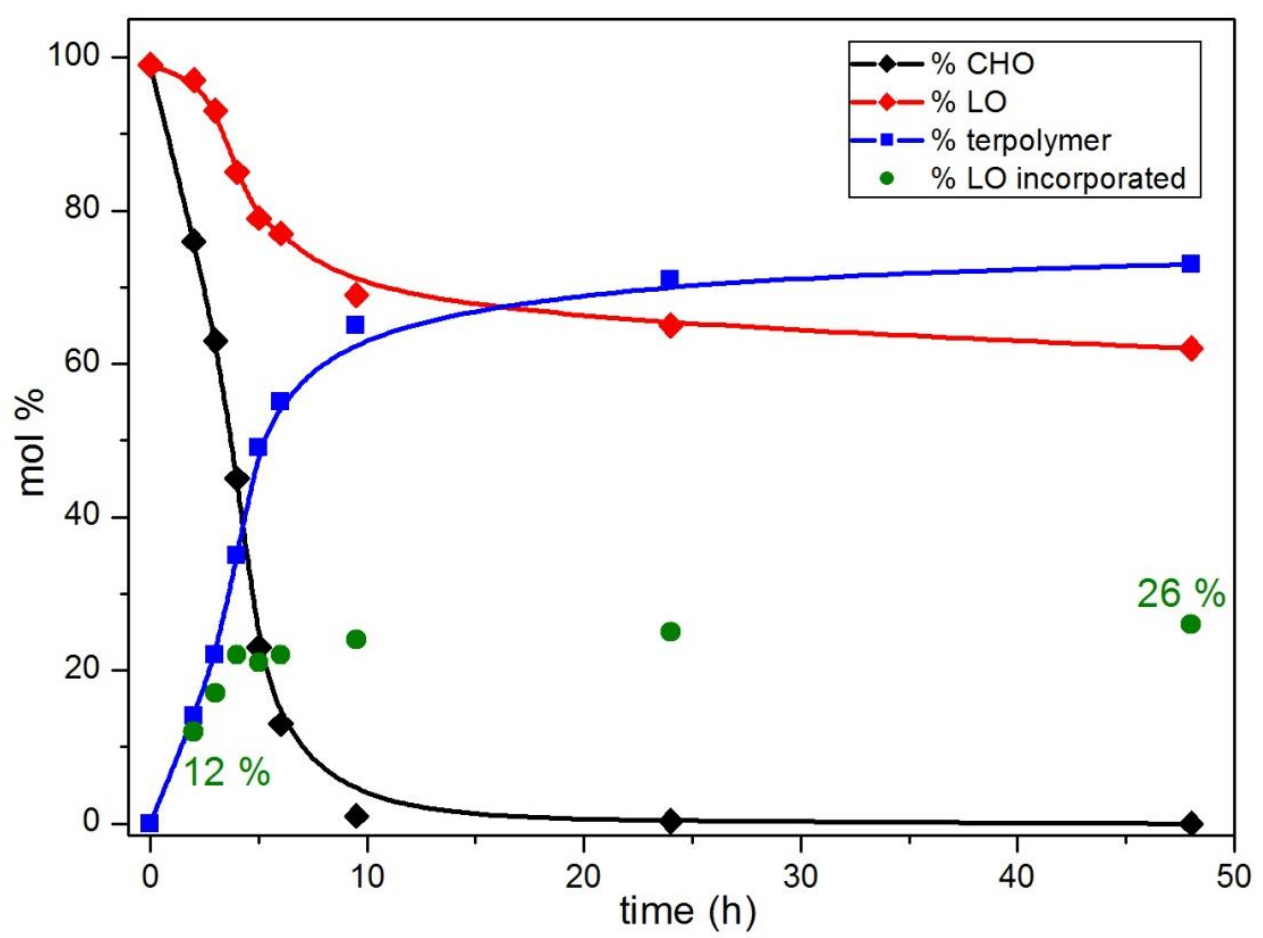

Figure 4. Kinetic profiles for the $\mathrm{CHO}$ and cis-LO (denoted as LO) consumption and the terpolymer formation. In green, the average \% of LO incorporation throughout the reaction. Reaction conditions: $\mathrm{CHO} / \mathrm{LO}\left(1: 1 \mathrm{ratio}, 3.5 \mathrm{mmol}\right.$ in total), $\mathbf{A l}^{\mathrm{Me}} 0.50 \mathrm{~mol} \%$, PPNCl $0.25 \mathrm{~mol}$ $\%, p\left(\mathrm{CO}_{2}\right)^{\circ}=15$ bar, neat, $40^{\circ} \mathrm{C}$. Polycarbonate conversion, and $\mathrm{CHO}$ and LO consumption were determined by ${ }^{1} \mathrm{H}$ NMR analysis of the crude reaction mixture using 1,4-dimethoxybenzene as internal standard. 
Notably, after a $10 \mathrm{~h}$ reaction time the overall reaction kinetics become rather slow likely as a consequence of the observed increasing viscosity of the mixture. It is thus clear that the terpolymer starts with a low content of incorporated LO monomer (12\%) but after $10 \mathrm{~h}$, when the CHO monomer conversion reaches around 95\% (Figure 4) this becomes significantly higher with $\mathrm{CHO} / \mathrm{LO}$ incorporation ratios $<0.5$ (for more details see Table S2, Supporting Information). Overall, this results in a terpolymer structure with a clear gradient in the pendant olefin groups.

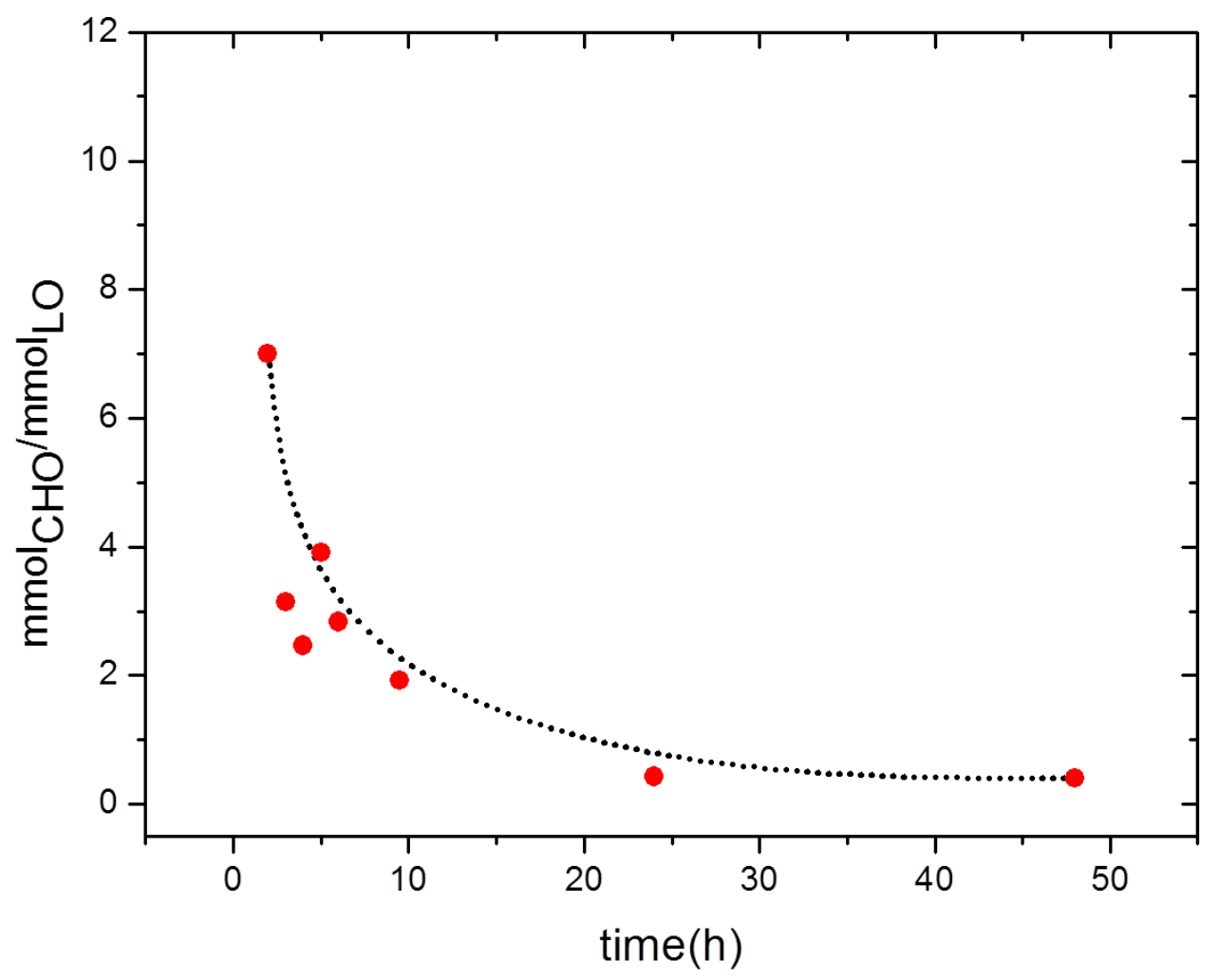

Figure 5. The change in the relative $\mathrm{CHO} / \mathrm{LO}$ incorporation kinetics in time for the terpolymerization process carried out at $40^{\circ} \mathrm{C}, 15$ bar and using 1:1 ratio of $\mathrm{CHO}$ and $\mathrm{LO}$ (3.5 mmol in total). 


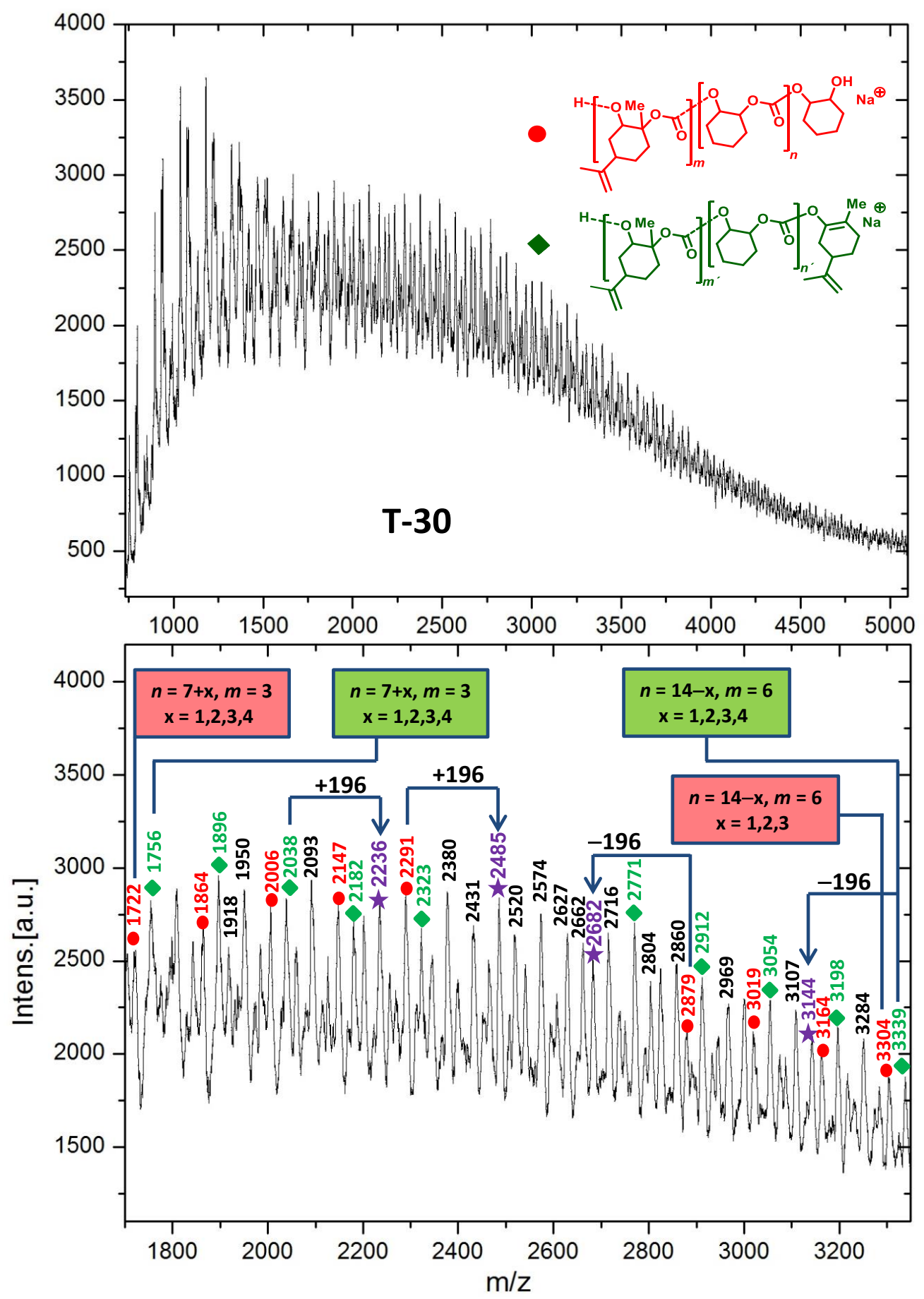

Figure 6. MALDI-TOF mass spectrum recorded for terpolymer T-30. The upper part shows the $1000-5000 \mathrm{~m} / \mathrm{z}$ region, whereas the lower part displays an enlarged portion between $1700-3400$ with some of the assigned peaks explained together with the most dominant end-groups observed. 
The terpolymer structures $\mathbf{T}-\boldsymbol{m}(\boldsymbol{m}=10,20,30$ or 40 ; Table 1 entries $2-5)$ were further investigated by MALDI-TOF mass spectrometry (Figure 6, and Supporting Information: Figures S53-S56) using similar conditions that were previously successfully used for $\mathrm{LO} / \mathrm{CO}_{2}$ copolymers. ${ }^{17}$ As a representative example the characteristics of $\mathbf{T - 3 0}$ (having an approximate $30 \%$ average of $\mathrm{LO} / \mathrm{CO}_{2}$ carbonate repeat units) are discussed here. ${ }^{24}$ The full trace (Figure 6 , upper part) and the selected, enlarged MALDI-TOF region $(m / z, 1700-3400)$ show a complicated combination of peaks. Despite this complexity, we were able to assign most of the peaks by considering (1) the preference for specific end-groups such as $-\mathrm{OH}$ and those having $\mathrm{H}_{2} \mathrm{O}$ eliminated (see terpolymer schematic insets at the top of Figure 6), ${ }^{25}$ and (2) the average $\%$ of LO incorporated in the terpolymer T-30 determined by ${ }^{1} \mathrm{H}$ NMR analysis. For instance, the peak at $m / z=1722$ could be readily assigned to a terpolymer based on seven $\mathrm{CHO} / \mathrm{CO}_{2}$ and three $\mathrm{LO} / \mathrm{CO}_{2}$ repeat units with a cyclohexanol end-group (calculated: $m / z=1721$ ), and subsequent incorporation of four $\mathrm{CHO} / \mathrm{CO}_{2}$ fragments $(\mathrm{m} / \mathrm{z}=1864,2006,2147$ and $2291 ; \Delta \mathrm{m}=142$, equal to a $\mathrm{CHO} / \mathrm{CO}_{2}$ repeat unit) was recognized. At various stages also the incorporation of new $\mathrm{LO} / \mathrm{CO}_{2}$ repeat units (196) was detected, for instance from $m / z=1722$ to 1918 and from $\mathrm{m} / z=$ 2291 to 2485 .

Apart from all the possible combinations of $n$ and $m$ (i.e., the ratio of $\mathrm{CHO} / \mathrm{CO}_{2} v s \mathrm{LO} / \mathrm{CO}_{2}$ repeat units for $\mathbf{T - 3 0}$ being an average of around 30\%), also the presence of different end-groups in these polymer distributions add to the complexity of the analysis. The peak at $\mathrm{m} / z=1756$ (calculated: 1757) was assigned to a terpolymer having the same $n / m$ ratio as found for the peak at $m / z=1722$ but with a different end-group based on a dehydrated LO fragment (upper part of Figure 6, green schematic inset). Propagation through consecutive $\mathrm{CHO} / \mathrm{CO}_{2}$ insertions is again easily recognized giving rise to polymers with increasing $n$ up to $11(\mathrm{~m} / z=2323$, calculated: 
2324), and the incorporation of $\mathrm{LO} / \mathrm{CO}_{2}$ repeat units can also be observed from, for instance, the peak at $m / z=2038(n=9, m=3)$ to give a polymer chain with increasing $\boldsymbol{m}(n=9, m=4: m / z=$ 2236, calculated: 2234).

In the higher mass region up to $m / z 3300$ similar type of distributions can be found, and the MALDI-TOF analysis therefore provides evidence for a complex mixture of polymer structures with distinct $n / m$ ratios, but more importantly it provides further proof for the early incorporation of the functional monomer LO into the polymer backbones. This corroborates with the kinetic profiles for $\mathrm{CHO}$ and $\mathrm{LO}$ consumption and the synthesis of terpolymeric structures with a clear gradient in the presence of the olefin groups from relatively low (12\% in the first $2 \mathrm{~h}$ ) to high $(>26 \%)$ at the end of the terpolymerization process.

Scheme 1. Synthesis of Cross-Linked Polymers through Radical-Initiated Thiol-Ene Click Chemistry. Here $\boldsymbol{m}$ Denotes the $\%$ of $\mathrm{LO} / \mathrm{CO}_{2}$ Repeat Units determined in the Different Terpolymers by ${ }^{1} \mathrm{H}$ NMR, whereas $\mathbf{T}-\boldsymbol{m}$ is the Terpolymer Substrate and CLP- $\boldsymbol{m}$ the CrossLinked Polymer.

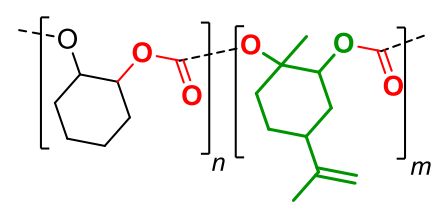

T-m

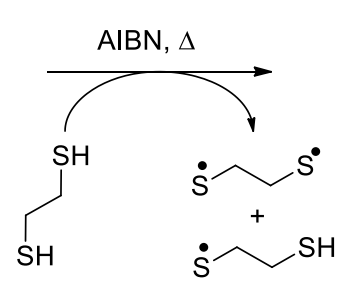

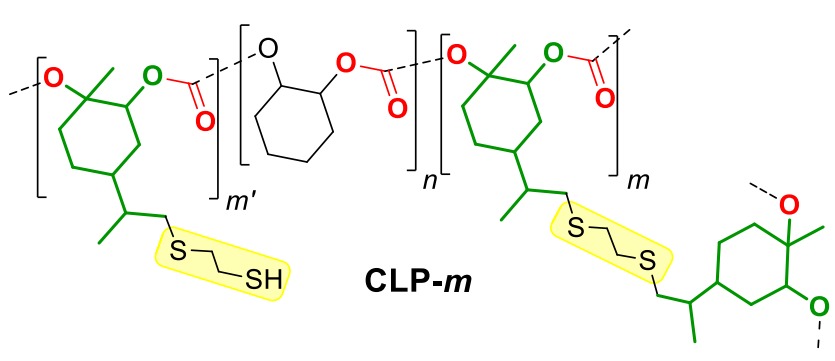

Terpolymer Cross-Linking Reactions. Having prepared a varied range of polycarbonates containing a controlled number of the functional monomer LO, we next turned our focus on post- 
polymerization modifications with the aim to improve their thermic properties. Since the pendent olefin groups are reasonably distributed throughout the polymer structures, we envisioned that cross-linking of the alkene units by thiol-ene chemistry would be an ideal strategy to prepare thermally more stable terpolymeric architectures. Thus, the partial bio-based terpolymers described in Table 1 were treated with 1,2-ethanedithiol in the presence of 2,2'-azobis(2methylpropionitrile) (AIBN) used as radical initiator in catalytic amounts ( 0.20 equiv. vs amount of olefin groups) in THF. The thiyl radicals generated by heating the reaction mixture to $70^{\circ} \mathrm{C}$ should attack the least hindered carbon center of the olefin unit (Scheme 1) and enable the connection of two separate $\mathrm{LO} / \mathrm{CO}_{2}$ repeat units either via intra- or inter-molecular pathways. It should be noted that the reaction stoichiometry is likely a decisive factor to control the ratio between mono- and di-reacted dithiol linkers which may influence the overall polymer properties.

The thiol-ene addition reaction leads to a gradual disappearance of the olefin groups in the polymer backbone, a change that could be easily followed and confirmed by IR and NMR spectroscopy (see Figures 7 and 8). For instance, evidence for thioether formation is provided by changes in the typical IR bands for the copolymer P-100 substrate located at $1645 \mathrm{~cm}^{-1}$ (C=C) and $887 / 837 \mathrm{~cm}^{-1}(\mathrm{C}=\mathrm{C}-\mathrm{H})$ that disappear in time (Figure 7).

${ }^{1} \mathrm{H}$ NMR analysis of T-20 treated with 1,2-ethanedithiol to give CLP-20 provided additional confirmation of olefin to thioether conversion in the polymer structure (Figure 8). For example, the appearance of a peak at $0.98 \mathrm{ppm}$ can be ascribed to thioether linkage formation causing a clear upfield shift of the methyl resonance of the incorporated LO unit (colored green; in the yellow sphere). Also, the presence of various peaks in the region 2.5-3.0 ppm supports the presence of thioether linkages. ${ }^{26}$ When a (larger) excess of 1,2-ethanedithiol is used, a peak with 
increasing intensity at $2.73 \mathrm{ppm}$ is noted which can be ascribed to the presence of cumulative amounts of mono-coupled dithiol species ( $c f$., presence of $-\mathrm{CH}_{2} \mathrm{SH}$ pendant fragments). In addition to these observation done by ${ }^{1} \mathrm{H}$ NMR, the disappearance of the quaternary and secondary carbon peaks pertinent to the olefin groups at 148.6 and $108.6 \mathrm{ppm}$, respectively, of T-20 was also noticed in the ${ }^{13} \mathrm{C}$ NMR (see Supporting Information: Figure S25).

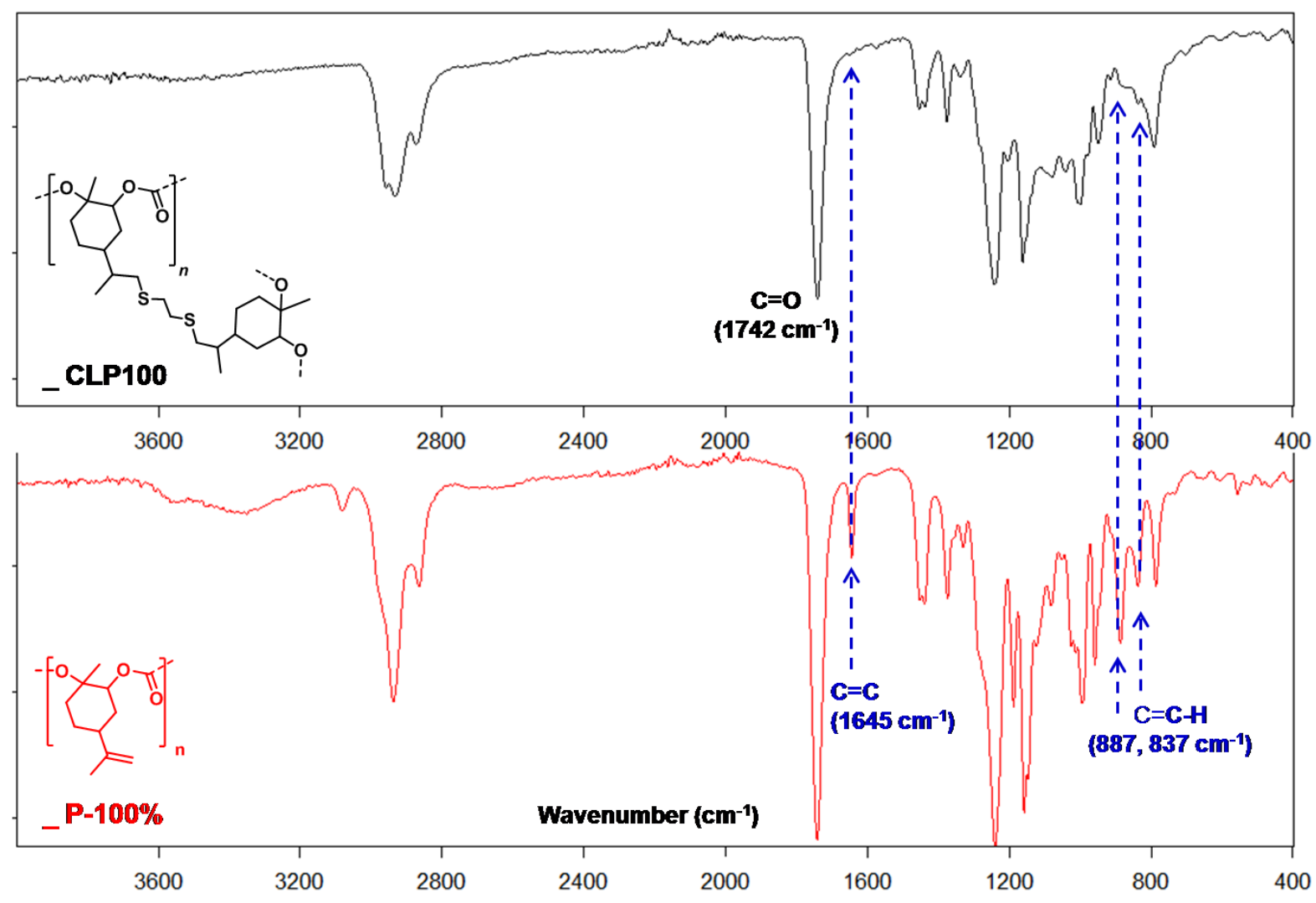

Figure 7. Comparative IR spectra for the copolymer P-100 and its corresponding cross-linked polymer CLP-100 (top) after the thiol-ene reaction. 

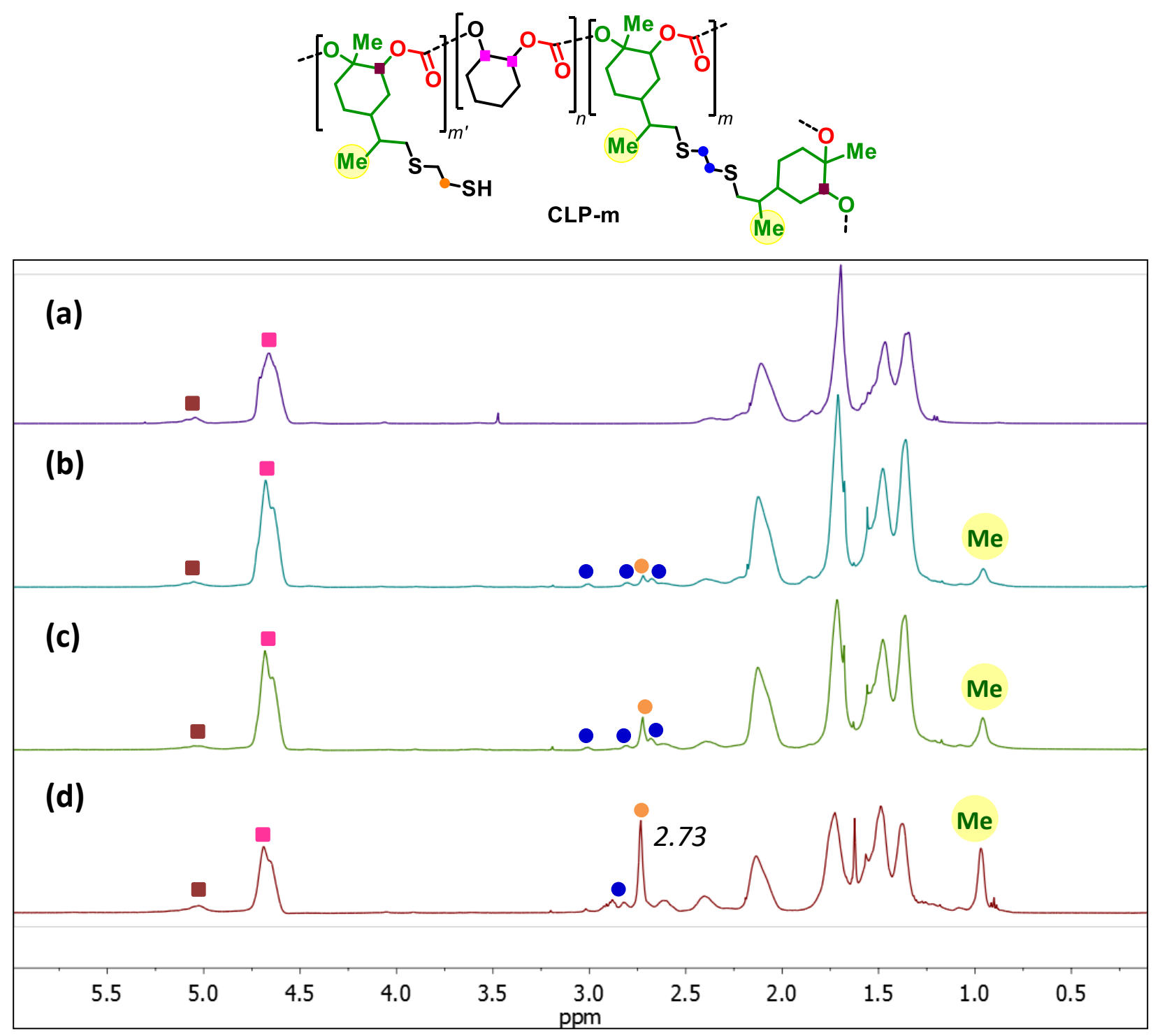

Figure 8. Comparative ${ }^{1} \mathrm{H}$ NMR spectra in $\mathrm{CDCl}_{3}$ at $25^{\circ} \mathrm{C}$ for: (a) isolated T-20 terpolymer, and (b-d) prepared cross-linked polymers CLP-20 using different 1,2-ethanedithiol molar equiv. in the cross-linking process: (b) 0.50 equiv., (c) 0.70 equiv. and (d) 1.5 equiv. The colored spheres represent the relevant protons attached to the indicated carbon centers in the schematic structure above. 
The optimal reaction conditions for the cross-linked polymer synthesis were then further examined with particular attention dedicated to influence of the cross-linking reagent on the polymer properties (Table 2). We performed a series of reactions with terpolymer T-20 and varied the concentration of 1,2-ethanedithiol. Moreover, reactions were performed under concentrated conditions to maximize intermolecular cross-linking (i.e., $1.0 \mathrm{~mL}$ of solvent per 150 mg of polymer substrate). The final product may incorporate thiol pendant groups ( $\boldsymbol{m}$ ' repeat units in Scheme 1). Indeed, we were able to detect such pendant groups by ${ }^{1} \mathrm{H}$ NMR spectroscopy (Figure 8) and their amount depended on the molar equiv. of 1,2-ethanedithiol used in the cross-linking process. Figure 8 suggest that the percentage of free, unreacted $-\mathrm{SH}$ groups should be minimal upon using a stoichiometric amount of dithiol reagent (i.e., 0.50 equiv.).

The post-modified CLP's were analyzed by differential scanning calorimetry (DSC) and thermogravimetric analysis (TGA). These thermal analyses showed that interconnected networks CLP-20a and CLP-20b derived after the cross-linking using an excess (> 0.50 molar equiv.) of dithiol reagent exhibited the lowest $T_{\mathrm{g}}$ and $T_{\mathrm{d}}$ values among the series (Table 2, entries 1 and 2 ). Conversely, the use of a stoichiometric amount of dithiol ( 0.50 equiv., entry 3$)$ resulted into the formation of a cross-linked polymer CLP-20c with better thermal properties. The reaction was then also carried out in the presence of a smaller than stochiometric amount of dithiol $(0.30$ equiv., entry 4). Under these conditions, the resulting CLP-20d did not show any improvement relative towards CLP-20c. Additionally, no observable reaction was noted under dithiol-free conditions in the presence of AIBN as radical initiator (entry 5, Table 2). From the combined results it can be concluded that the use of a stoichiometric amount of dithiol produces a crosslinked polymer with optimized thermal features. 
Table 2. Synthesis of Cross-Linked Polymers via Thiol-Ene Click Chemistry varying the Amount of Dithiol and using Terpolymer T-20. ${ }^{a, b}$

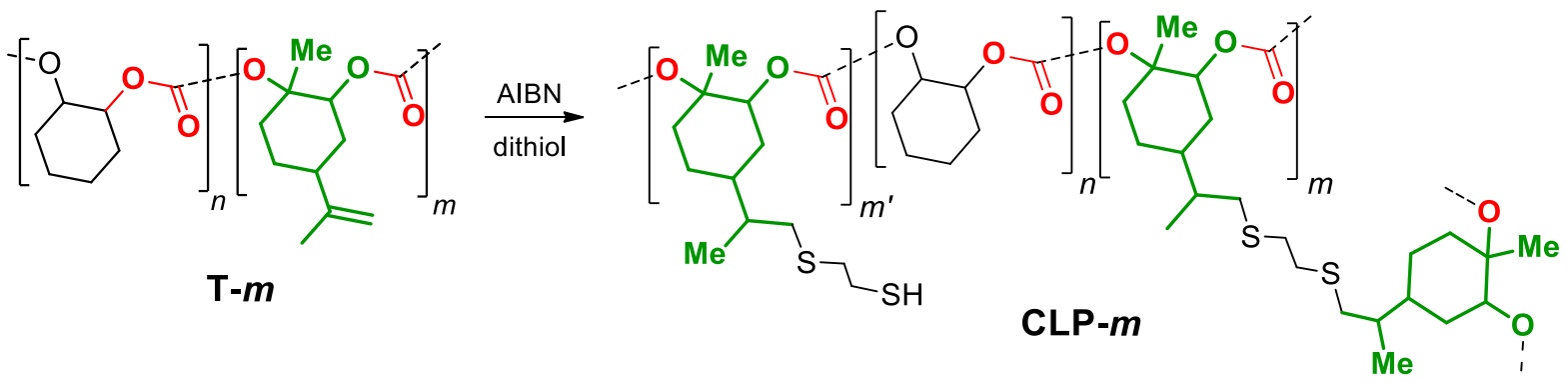

\begin{tabular}{ccccccc}
\hline Entry & ${\text { dithiol (equiv })^{c}}^{c}$ & $\mathbf{C L P}^{d}$ & $\boldsymbol{T}_{\mathbf{g}}\left({ }^{\mathbf{o}} \mathbf{C}\right)^{e}$ & $\Delta \boldsymbol{T}_{\mathbf{g}}\left({ }^{\mathbf{o}} \mathbf{C}\right)^{f}$ & $\boldsymbol{T}_{\mathbf{d}}\left({ }^{\mathbf{0}} \mathbf{C}\right)^{g}$ & $\Delta \boldsymbol{T}_{\mathbf{d}}\left({ }^{\mathbf{o}} \mathbf{C}\right)^{h}$ \\
\hline 1 & 1.5 & $\mathbf{C L P - 2 0 a}$ & 107 & 7 & 264 & 16 \\
2 & 0.70 & $\mathbf{C L P - 2 0 b}$ & 109 & 6 & 272 & 24 \\
3 & 0.50 & CLP-20c & 118 & 18 & 279 & 31 \\
4 & 0.30 & CLP-20d & 112 & 9 & 272 & 24 \\
5 & 0 & - & 100 & - & 248 & -
\end{tabular}

${ }^{a}$ Reaction conditions: $150 \mathrm{mg}$ of terpolymer T-20, AIBN (0.20 equiv. $v s$ olefin groups), $12 \mathrm{~h}$, THF (1.0 mL), $70^{\circ} \mathrm{C} .{ }^{b} T_{\mathrm{g}}$ and $T_{\mathrm{d}}$ values for the sample $\mathbf{T}-20$ used in these studies were $100^{\circ} \mathrm{C}$ and $248^{\circ} \mathrm{C}$, respectively. ${ }^{c}$ Equivalents of 1,2 -ethanedithiol used $v s$ amount of olefin groups. ${ }^{d}$ Crosslinked polymer (CLP) containing a maximum $\boldsymbol{m} \%$ of linkages. ${ }^{e}$ Determined by differential scanning calorimetry (DSC). ${ }^{f} \Delta T_{\mathrm{g}}$ is the difference between the $T_{\mathrm{g}}$ of the cross-linked polymer and the T-20 terpolymer substrate. ${ }^{g}$ Decomposition temperature $\left(T_{\mathrm{d}}\right)$ determined by thermogravimetric analysis (TGA) at $10 \%$ weight loss of the polymer. ${ }^{h} \Delta T_{\mathrm{d}}$ is the difference between $T_{\mathrm{d}}$ values of the cross-linked polymer and the T-20 terpolymer substrate.

With these optimized conditions ( 0.50 equiv. dithiol, 0.20 equiv. of $\mathrm{AIBN}, 12 \mathrm{~h}, 70^{\circ} \mathrm{C}, \mathrm{THF}$ ), we then further investigated the formation of cross-linked terpolymers with different amount of olefin groups (T- $\boldsymbol{m} ; \boldsymbol{m}=10,20,30,40 \%$ and P-100; Table 3$).{ }^{27}$ For the CLP's derived from T10 and T-20 we observed the quantitative conversion of the olefin groups and formation of soluble networks CLP-10 and CLP-20. ${ }^{28}$ However, a further increase in reactive olefin groups in the ter/copolymer substrate (T-30, T-40 and $\mathbf{P - 1 0 0})$ resulted in the formation of materials that 
are virtually insoluble in organic solvents. From Table 3 it can be deduced that the cross-linking reaction had a pronounced positive effect on the thermal stability of the resultant CLP polymers increasing the decomposition temperatures $\left(T_{\mathrm{d}}\right)$ up to $54^{\circ} \mathrm{C}$ (for CLP-30, entry 3 ). Interestingly, this cross-linking process also enhanced the glass transition temperature and the CLP's exhibited single $T_{\mathrm{g}}{ }^{\prime} \mathrm{s}$ from $116^{\circ} \mathrm{C}\left(\mathbf{C L P}-30\right.$, entry 3) up to $150^{\circ} \mathrm{C}$ (CLP-100, entry 5). As expected, the $T_{\mathrm{g}}$ values increased as a function of the amount of cross-linkable olefin groups with CLP-40 (entry 4) and CLP-100 (entry 5) displaying the highest $T_{\mathrm{g}}$. In the latter case, the $T_{\mathrm{g}}$ increased from 73 to $150^{\circ} \mathrm{C}\left(\Delta T_{\mathrm{g}}=77^{\circ} \mathrm{C}\right)$ despite the rather modest molecular weight $\left(M_{\mathrm{n}}=3.69 \mathrm{~kg} / \mathrm{mol}\right.$; Table 1 , entry 6) of the copolymer substrate $\mathbf{P - 1 0 0}$. Copolymer $\mathbf{P}-\mathbf{1 0 0}$ was also reacted with an excess of dithiol (Table 3, entry 6) towards the formation of a CLP with a larger amount of unreacted thiol groups as observed for T-20 $(c f$., formation of CLP-20a and CLP20b, entries 1 and 2 in Table 2). The resulting cross-linked polymer CLP-100a showed a much lower $T_{\mathrm{g}}$ and also a lower $T_{\mathrm{d}}$ values than obtained for CLP-100 thus supporting the view that stoichiometric amounts $(0.50$ molar equiv.) of the dithiol reagent are also ideal for converting polymers substrates with higher cross-linkable olefin densities into thermally more stable materials. Under more dilute conditions (entry 7), the CLP-100b produced from T-100 was fully soluble and showed virtually no change in the $T_{\mathrm{g}}$ value. This indicates that most of the olefin groups had either reacted in an intramolecular fashion and/or had led to a considerable concentration of unreacted mono-thiol groups in the polymer backbone. GPC analysis of this post-modified polymer CLP-100b showed a considerable increase in molecular weight $\left(M_{\mathrm{n}}=9.51 \mathrm{~kg} / \mathrm{mol}\right)$ and polydispersity $(\nexists=3.15)$ compared with P-100 $\left(M_{\mathrm{n}}=3.69 \mathrm{~kg} / \mathrm{mol}, \oslash=1.34\right)$. Thus, the cross-linking process carried out under diluted conditions favors to a larger extent olefin functionalization and/or introduction of intramolecular linkages though some level of intermolecular cross-linking is maintained. 
Table 3. Synthesis of Various Cross-Linked Polymers (CLPs) via Thiol-Ene Click Chemistry. ${ }^{a}$

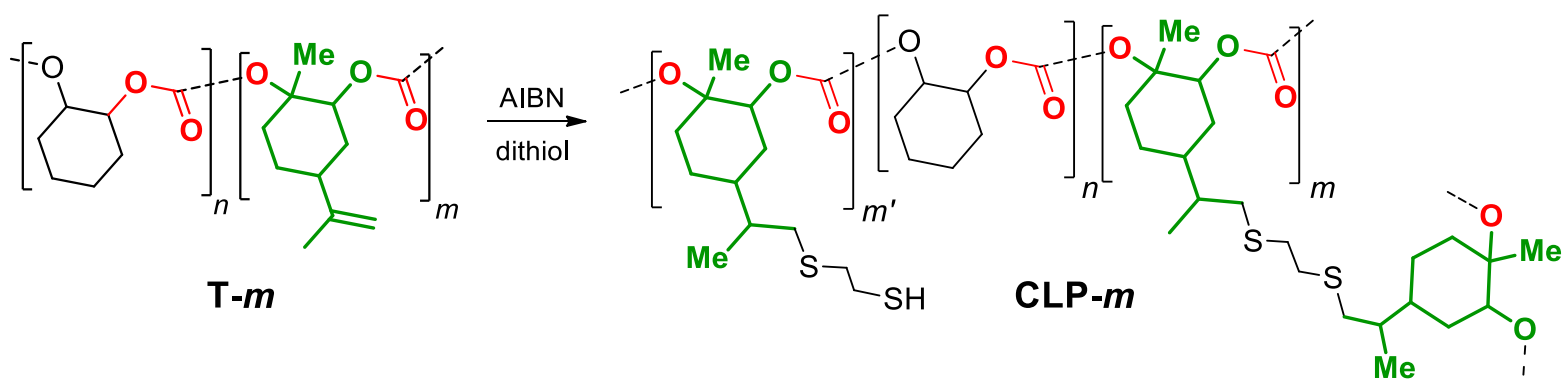

\begin{tabular}{ccccccccc}
\hline Entry & $\mathbf{T}-\boldsymbol{m}^{b}$ & $\begin{array}{c}\boldsymbol{T}_{\mathbf{g}} \\
\left({ }^{\circ} \mathbf{C}\right)^{c}\end{array}$ & $\begin{array}{c}\boldsymbol{T}_{\mathbf{d}} \\
\left({ }^{\circ} \mathbf{C}\right)^{d}\end{array}$ & $\mathbf{C L P}-\mathbf{m}^{e}$ & $\begin{array}{c}\boldsymbol{T}_{\mathbf{g}} \\
\left({ }^{\circ} \mathbf{C}\right)^{c}\end{array}$ & $\begin{array}{c}\Delta \boldsymbol{T}_{\mathbf{g}} \\
\left({ }^{\circ} \mathbf{C}\right)^{f}\end{array}$ & $\begin{array}{c}\boldsymbol{T}_{\mathbf{d}} \\
\left({ }^{\circ} \mathbf{C}\right)^{d}\end{array}$ & $\begin{array}{c}\Delta \boldsymbol{T}_{\mathbf{d}} \\
\left({ }^{\circ} \mathbf{C}\right)^{g}\end{array}$ \\
\hline 1 & $\mathbf{T}-10$ & 102 & 263 & $\mathbf{C L P}-10$ & 117 & 15 & 282 & 19 \\
2 & $\mathbf{T}-20$ & 100 & 248 & $\mathbf{C L P}-20$ & 118 & 18 & 279 & 31 \\
3 & $\mathbf{T}-30$ & 100 & 210 & $\mathbf{C L P}-30$ & 116 & 16 & 264 & 54 \\
4 & $\mathbf{T}-40$ & 97 & 228 & $\mathbf{C L P}-40$ & 119 & 22 & 256 & 28 \\
5 & $\mathbf{P}-100$ & 73 & 226 & $\mathbf{C L P}-100$ & 150 & 77 & 245 & 19 \\
$6^{h}$ & $\mathbf{P}-100$ & 73 & 226 & $\mathbf{C L P}-100 a$ & 83 & 10 & 239 & 13 \\
$7^{i}$ & $\mathbf{P}-100$ & 75 & 226 & $\mathbf{C L P - 1 0 0 b}$ & 74 & 0 & 233 & 7 \\
\hline
\end{tabular}

${ }^{a}$ Reaction conditions: $150 \mathrm{mg}$ of terpolymer, 1,2-ethanedithiol (0.50 equiv. $v s$ olefin groups), AIBN (0.20 equiv. $v s$ olefin groups), $12 \mathrm{~h}, 70^{\circ} \mathrm{C} .{ }^{b}$ Terpolymer containing $\boldsymbol{m} \%$ of LO monomer incorporated. ${ }^{c}$ Determined by differential scanning calorimetry (DSC). ${ }^{d}$ Decomposition temperature $\left(T_{\mathrm{d}}\right)$ determined by thermogravimetric analysis (TGA) at $10 \%$ weight loss of the polymer. ${ }^{e}$ Cross-linked polymer (CLP) containing a maximum of $\boldsymbol{m} \%$ of linkages. ${ }^{f} \Delta T_{\mathrm{g}}$ is the difference between the $T_{\mathrm{g}}$ values of the cross-linked polymer CLP and the terpolymer T-m substrate. ${ }^{g} \Delta T_{\mathrm{d}}$ is the difference between the $T_{\mathrm{d}}$ values of the cross-linked polymer CLP and the terpolymer T-m substrate. ${ }^{h}$ Using 1.0 equiv. of 1,2-ethanedithiol $v s$ olefin groups. ${ }^{i}$ Using $20 \mathrm{~mL}$ of THF as solvent.

The increase of the rigidity in the CLP's with increasing cross-linking density could be observed by DSC analysis as shown in Figure 9. The CLP's having a higher amount of linker units (i.e., CLP-30, CLP-40 and CLP-100) displayed more flattened glass transitions and this 
behavior may be expected of highly rigid ${ }^{29}$ and intermolecular connected polymer chains, in line with the poorer solubility observed for the CLP's.

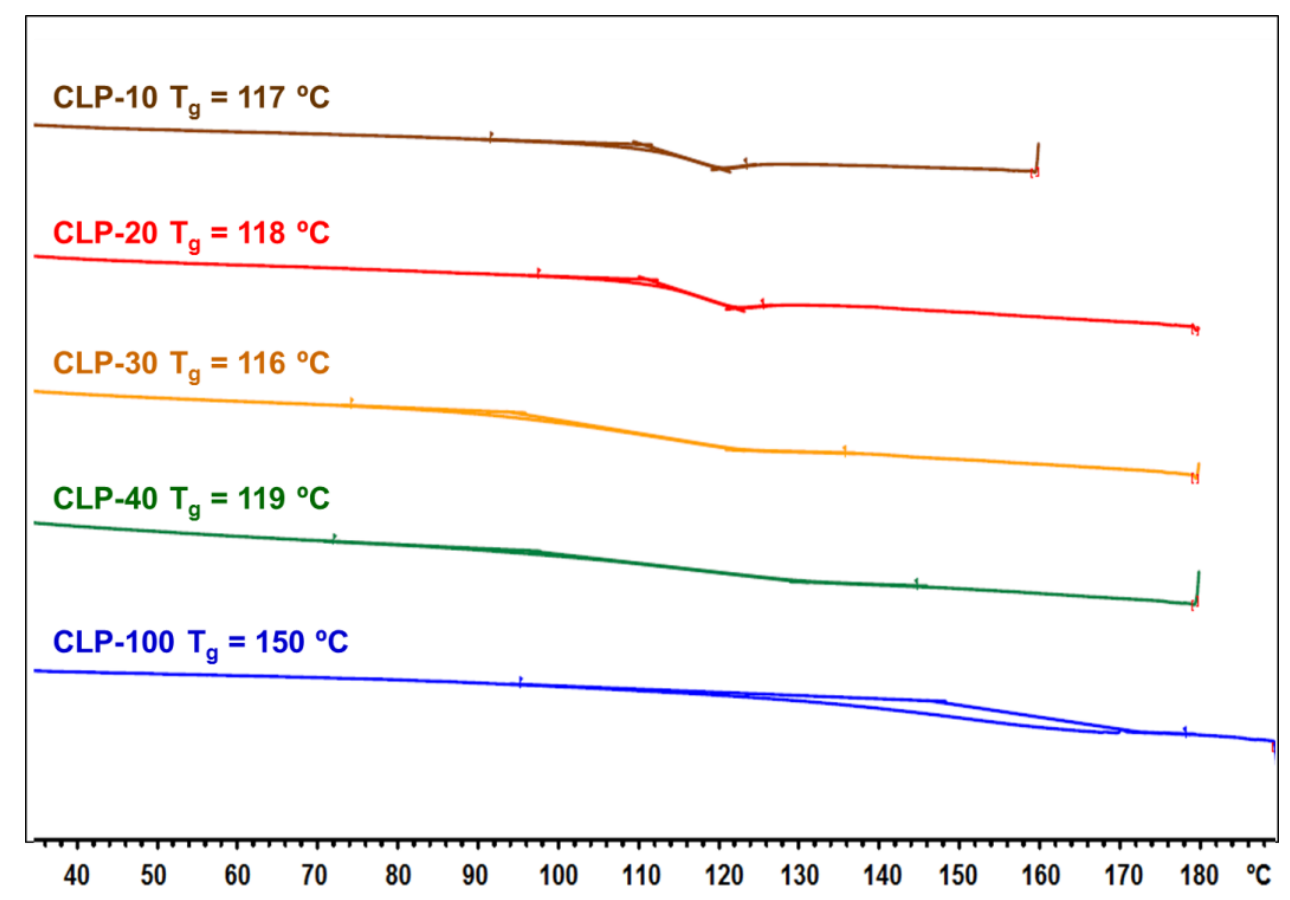

Figure 9. Selected region of the differential scanning calorimetry (DSC) analysis of CLP-10, CLP-20, CLP-30, CLP-40 and CLP-100. The heating rate was $10^{\circ} \mathrm{C} / \mathrm{min}$. In each case, the trace of the second cycle is shown. 


\section{CONCLUSION}

In summary, we describe here the first catalytic process towards the formation of terpolymers based on the naturally occurring terpene limonene oxide (LO) with a controlled number of functional repeat units. The control over the number of olefin units enables the post-modification of these terpolymers through thiol-ene chemistry providing materials with improved thermal properties exhibiting much higher $T_{\mathrm{g}}$ and $T_{\mathrm{d}}$ values than their corresponding terpolymer precursors. These new cross-linked polymers (CLP's) thus provide a partial bio-based alternative for those systems that are derived from fossil fuel based feed stocks only. The combined analytical data (IR, NMR, TGA, DSC and GPC) shows good control in the radical initiated thiol-ene cross-linking process when stoichiometric amount of dithiol reagent $(0.50$ equiv. vs olefin groups) is utilized and emphasizes the importance of the appropriate reaction stoichiometry, and the influence of the amount of functional groups present in the ter/copolymer backbone enabling the synthesis of new cross-linked materials with $T_{\mathrm{g}}{ }^{\prime} \mathrm{s}$ of up to $150^{\circ} \mathrm{C}$. Due to the vast increase in bio-renewable materials in industry and academic settings, our current focus is now on the design of new and stable (partially) bio-based co- and terpolymers with different architectures and novel properties using terpenes as accessible and modular scaffolds. ${ }^{30}$

\section{ASSOCIATED CONTENT}

Supporting Information. Further experimental details, spectra of isolated polymer samples (NMR and IR), and relevant GPC, MALDI-TOF-MS, DSC and TGA traces. This material is available free of charge via the Internet at http://pubs.acs.org. 


\section{AUTHOR INFORMATION}

\section{Corresponding Author}

akleij@iciq.es

Notes

The authors declare no competing financial interest.

\section{ACKNOWLEDGMENTS}

We acknowledge financial support from ICIQ, ICREA, the Spanish MINECO (project CTQ2014-60419-R) and the Severo Ochoa Excellence Accreditation 2014-2018 through project SEV-2013-0319. C. M. is grateful to the Marie Curie COFUND action from the European Commission for co-financing a postdoctoral fellowship. The authors also thank Dr. Marta Giménez and Cristina Rivero for assistance with high pressure experiments and Dr. Noemí Cabello for the MALDI-TOF MS analyses. Simona Curreli, María José Hueso and Marta Serrano are thanked for their help regarding the thermal analyses (TGA/DSC). 


\section{REFERENCES}

(1) (a) Williams, C. K.; M. A. Hillmyer, Polymers from Renewable Resources: A Perspective for a Special Issue of Polymer Reviews. Polym. Rev. 2008, 48, 1-10. (b) Ciriminna, R.; LomeliRodriguez, M.; Demma Carà, P.; Lopez-Sanchez, J. A.; Pagliaro, M. Limonene: a Versatile Chemical of the Bioeconomy. Chem. Commun. 2014, 50, 15288-15296. (c) Ishimoto, K.; Arimoto, M.; Okuda, T.; Yamaguchi, S.; Aso, Y.; Ohara, H.; Kobayashi, S.; Ishii, M.; Morita, K.; Yamashita, H.; Yabuuchi, N. Biobased Polymer System: Mini-Emulsion of Poly(alkyl methacrylate-graft-lactic acid)s. Biomacromolecules 2012, 13, 3757-3768.

(2) (a) Liu, Q.; Wu, L.; Jackstell, R.; Beller, M. Using Carbon Dioxide as a Building Block in Organic Synthesis. Nat. Commun. 2015, 6, 5933. (b) Kielland, N.; Whiteoak, C. J.; Kleij, A. W. Stereoselective Synthesis with Carbon Dioxide. Adv. Synth. Catal. 2013, 355, 2115-2138. (c) Zhang, L.; Hou, Z. N-Heterocyclic Carbene (NHC)-Copper-Catalysed Transformations of Carbon Dioxide. Chem. Sci. 2013, 4, 3395-3403.

(3) (a) Hearon, K.; Nash, L. D.; Rodriguez, J. N., Lonnecker, A. T.; Raymond, J. E.; Wilson, T.

S.; Wooley, K. L.; Maitland, D. J. A High-Performance Recycling Solution for Polystyrene Achieved by the Synthesis of Renewable Poly(thioether) Networks Derived from d-Limonene. Adv. Mater. 2014, 26, 1552-1558. (b) Hong, M.; Chen, E. Y.-X. Completely Recyclable Biopolymers with Linear and Cyclic Topologies via Ring-Opening Polymerization of $\gamma$ Butyrolactone. Nat. Chem. 2016, 8, 42-49. (c) Miyaji, H., Satoh, K.; Kamigaito, M. Bio-Based Polyketones by Selective Ring-Opening Radical Polymerization of $\alpha$-Pinene-Derived Pinocarvone. Angew. Chem. Int. Ed. 2016, 53, 1372-1376. 
(4) (a) Inoue, S.; Koinuma, H.; Tsuruta, T. Copolymerization of Carbon Dioxide and Epoxide. J. Polym. Sci. Part B: Polym. Lett. 1969, 7, 287-292. (b) Nozaki, K. Asymmetric Catalytic Synthesis of Polyketones and Polycarbonates. Pure Appl. Chem. 2004, 76, 541-546. (c) Coates, G. W.; Moore, D. R. Discrete Metal-Based Catalysts for the Copolymerization of $\mathrm{CO}_{2}$ and Epoxides: Discovery, Reactivity, Optimization, and Mechanism. Angew. Chem. Int. Ed. 2004, 116, 6784-6806. (d) Darensbourg, D. J. Making Plastics from Carbon Dioxide. Salen Metal Complexes as Catalysts for the Production of Polycarbonates from Epoxides and $\mathrm{CO}_{2}$. Chem. Rev. 2007, 107, 2388-2410. (e) Kember, M. R.; Buchard, A; Williams, C. K. Catalysts for $\mathrm{CO}_{2}$ /Epoxide Copolymerization. Chem. Commun. 2011, 47, 141-163. (f) Klaus, S.; Lehenmeier, M. W.; Anderson, C. E.; Rieger, B. Recent Advances in $\mathrm{CO}_{2}$ /Epoxide Copolymerization - New Strategies and Cooperative Mechanisms. Coord. Chem. Rev. 2011, 255, 1460-1479. (g) Lu, X.B.; Darensbourg, D. J. Cobalt Catalysts for the Coupling of $\mathrm{CO}_{2}$ and Epoxides to provide Polycarbonates and Cyclic Carbonates. Chem. Soc. Rev. 2012, 41, 1462-1484. (h) Lu, X.-B.; Ren, W.-M.; Wu, G.-P. $\mathrm{CO}_{2}$ Copolymers from Epoxides: Catalyst Activity, Product Selectivity, and Stereochemistry Control. Acc. Chem. Res. 2012, 45, 1721-1735. (i) Childers, M. I.; Longo, J. M.; Van Zee, N. J.; LaPointe, A. M.; Coates, G. W. Stereoselective Epoxide Polymerization and Copolymerization. Chem. Rev. 2014, 114, 8129-8152. (j) Aresta, M.; Dibenedetto, A.; Angelini, A. Catalysis for the Valorization of Exhaust Carbon: from $\mathrm{CO}_{2}$ to Chemicals, Materials, and Fuels. Technological Use of $\mathrm{CO}_{2}$. Chem. Rev. 2014, 114, 1709-1742. (h) Devaine-Pressing, K.; Dawe, L. N.; Kozak, C. M. Cyclohexene Oxide/Carbon Dioxide Copolymerization by Chromium(III) Aminobis(phenolato) Complexes and MALDI-TOF MS Analysis of the Polycarbonates. Polym. Chem. 2015, 6, 6305-6315. (i) Chen, H.; Dawe, L. N.; Kozak, C. M. Chromium(III) Amine-bis(phenolate) Complexes as Catalysts for 
Copolymerization of Cyclohexene Oxide and $\mathrm{CO}_{2}$. Catal. Sci. Technol. 2014, 4, 1547-1555. (j)

Nakano, K.; Kobayashi, K.; Ohkawara, T.; Imoto, H.; Nozaki, K. Copolymerization of Epoxides with Carbon Dioxide Catalyzed by Iron-Corrole Complexes: Synthesis of a Crystalline Copolymer. J. Am. Chem. Soc. 2013, 135, 8456-8459.

(5) (a) Byrne, C. M.; Allen; S. D.; Lobkovsky, E. B.; Coates, G. W. Alternating Copolymerization of Limonene Oxide and Carbon Dioxide. J. Am. Chem. Soc. 2004, 126, 11404-11405. (b) Auriemma, F.; de Rosa, C.; Di Caprio, M. R.; di Girolamo, R.; Chadwick Ellis, W.; Coates, G. W. Stereocomplexed Poly(Limonene Carbonate): A Unique Example of the Cocrystallization of Amorphous Enantiomeric Polymers. Angew. Chem. Int. Ed. 2015, 54, 12151218; Angew. Chem. 2015, 127, 1231-1234. (c) Hauenstein, O.; Reiter, M.; Agarwal, S.; Rieger, B.; Greiner, A. Bio-based Polycarbonate from Limonene Oxide and $\mathrm{CO}_{2}$ with High Molecular Weight, Excellent Thermal Resistance, Hardness and Transparency. Green Chem., 2016, 18, $760-770$.

(6) Zhang, Y.; Zhang, X.; Wei, R.; Du, B.; Fan, Z.; Qi, G. Synthesis of Fully Alternating Polycarbonate with Low Tg from Carbon Dioxide and Bio-based Fatty Acid. RSC Adv. 2014, 4, $36183-36188$.

(7) (a) Nakano, K.; Kamada, T.; Nozaki, K. Selective Formation of Polycarbonate over Cyclic Carbonate: Copolymerization of Epoxides with Carbon Dioxide Catalyzed by a Cobalt(III) Complex with a Piperidinium End-Capping Arm. Angew. Chem. Int. Ed. 2006, 45, 7274-7277. (b) Kim, J. G.; Cowman, C. D.; LaPointe, A. M.; Wiesner, U.; Coates, G. W. Tailored Living Block Copolymerization: Multiblock Poly(cyclohexene carbonate)s with Sequence Control. Macromolecules 2011, 44, 1110-1113. (c) Kim, J. G.; Coates, G. W. Synthesis and 
Polymerization of Norbornenyl-Terminated Multiblock Poly(cyclohexene carbonate)s: A Consecutive Ring-Opening Polymerization Route to Multisegmented Graft Polycarbonates. Macromolecules 2012, 45, 7878-7883.

(8) Wang, Y.; Fan, J.; Darensbourg, D. J. Construction of Versatile and Functional Nanostructures Derived from $\mathrm{CO}_{2}$-based Polycarbonates. Angew. Chem. Int. Ed. 2015, 54, $10206-10210$.

(9) Cherian, A. E.; Sun, F. C.; Sheiko, S. S.; Coates, G. W. Formation of Nanoparticles by Intramolecular Cross-Linking: Following the Reaction Progress of Single Polymer Chains by Atomic Force Microscopy. J. Am. Chem. Soc. 2007, 129, 11350-11351.

(10) (a) Hilf, J.; Frey, H. Propargyl-Functional Aliphatic Polycarbonate Obtained from Carbon Dioxide and Glycidyl Propargyl Ether. Macromol. Rapid Commun. 2013, 1395-1400. (b) Darensbourg, D. J.; Tsai, F.-T. Post-Polymerization Functionalization of Copolymers Produced from Carbon Dioxide and 2-Vinyloxirane: Amphiphilic/Water-Soluble $\mathrm{CO}_{2}$-Based Polycarbonates. Macromolecules 2014, 47, 3806-3813.

(11) Winkler, M.; Romain, C.; Meier, M. A. R., Williams, C. K. Renewable Polycarbonates and Polyesters from 1,4-Cyclohexadiene. Green Chem. 2015, 17, 300-306.

(12) (a) Geschwind, J.; Wurm, F.; Frey, H. From $\mathrm{CO}_{2}$-Based Multifunctional Polycarbonates With a Controlled Number of Functional Groups to Graft Polymers. Macromol. Chem. Phys. 2013, 214, 892-901. (b) Darensbourg, D. J.; Chung, W.-C.; Arp, C. J.; Tsai, F.-T.; Kyran, S. J. Copolymerization and Cycloaddition Products Derived from Coupling Reactions of 1,2-Epoxy- 
4-cyclohexene and Carbon Dioxide. Postpolymerization Functionalization via Thiol-ene Click Reactions. Macromolecules 2014, 47, 7347-7353.

(13) (a) Ryu, D. Y.; Shin, K.; Drockenmuller, E.; Hawker, C. J.; Russell, T. P. A Generalized Approach to the Modification of Solid Surfaces. Science 2005, 308, 236-239. (b) Spruell, J. M.; Wolffs, M.; Leibfarth, F. A.; Stahl, B. C.; Heo, J; Connal, L. A.; Hu, J.; Hawker, C. J Reactive, Multifunctional Polymer Films through Thermal Cross-linking of Orthogonal Click Groups. $J$. Am. Chem. Soc. 2011, 133, 16698-16706.

(14) Mavila, S.; Eivgi, O.; Berkovich, I.; Lemcoff, N. G. Intramolecular Cross-Linking Methodologies for the Synthesis of Polymer Nanoparticles. Chem. Rev. 2016, 116, 878-961.

(15) Darensbourg, D. J.; Wang, Y. Terpolymerization of Propylene Oxide and Vinyl Oxides with $\mathrm{CO}_{2}$ : Copolymer Cross-linking and Surface Modification via Thiol-ene Click Chemistry. Polym. Chem. 2015, 6, 1768-1776.

(16) Taherimerhr, M.; Cardoso Costa Serta, J. P.; Kleij, A. W., Whiteoak, C. J.; Pescarmona, P. P. New Iron Pyridylamino-Bis(Phenolate) Catalyst for Converting $\mathrm{CO}_{2}$ into Cyclic Carbonates and Cross-Linked Polycarbonates. ChemSusChem 2015, 8, 1034-1042.

(17) Peña Carrodeguas, L.; González-Fabra, J.; Castro-Gómez, F.; Bo, C.; Kleij, A. W. Al(III)Catalysed Formation of Poly(limonene)carbonate: DFT Analysis of the Origin of Stereoregularity. Chem. Eur. J. 2015, 21,6115-6122. 
(18) Steiner, D.; Ivison, L.; Goralski, C. T.; Appell, R. B.; Gojkovic, J. R.; Singaram, B. A facile and efficient Method for the Kinetic Separation of Commercially Available Cis- and Trans-limonene Epoxide. Tetrahedron Asymmetry 2002, 13, 2359-2363.

(19) (a) A. Chandrasekaran, R. O. Day, R. R. Holmes, A New Class of Silatranes: Structure and Dynamic NMR Behavior. J. Am. Chem. Soc. 2000, 122, 1066-1072. (b) C. J. Whiteoak, N. Kielland, V. Laserna, E. C. Escudero-Adán, E. Martin, A. W. Kleij, A Powerful Aluminum Catalyst for the Synthesis of Highly Functional Organic Carbonates. J. Am. Chem. Soc. 2013, $135,1228-1231$.

(20) Note that the molecular weight of the polymer substrate used in the cross-linking reactions was based on the average weight of the repeating units. For instance, the molecular weight of the repeat unit of $\mathbf{T}-10$ was $90 \%$ of the mass of the $\mathrm{CHO} / \mathrm{CO}_{2}$ repeat unit and $10 \%$ of the mass calculated for the $\mathrm{LO} / \mathrm{CO}_{2}$ repeat unit.

(21) Table S3 in the Supporting Information shows the results for several terpolymerization experiments and demonstrates the reproducible character of these processes.

(22) (a) Jeske, C. R.; Rowley, J. M.; Coates, G. W. Pre-rate-determining Selectivity in the Terpolymerization of Epoxides, Cyclic Anhydrides, and $\mathrm{CO}_{2}$ : a One-step Route to Diblock Copolymers. Angew. Chem. Int. Ed. 2008, 47, 6041-6044. (b) Darensbourg, D. J.; Poland, R. R.; Escobedo, C. Kinetic Studies of the Alternating Copolymerization of Cyclic Acid Anhydrides and Epoxides, and the Terpolymerization of Cyclic Acid Anhydrides, Epoxides, and $\mathrm{CO}_{2}$ Catalyzed by (salen)CrIII Cl. Macromolecules 2012, 45, 2242-2248. (c) Romain, C.; Williams, C. K. Chemoselective Polymerization Control: From Mixed-Monomer Feedstock to Copolymers. 
Angew. Chem. 2014, 126, 1633-1636. (d) Zhu, Y.; Romain, C.; Williams, C. K. Selective Polymerization Catalysis: Controlling the Metal Chain End Group to Prepare Block Copolyesters. J. Am. Chem. Soc. 2015, 137, 12179-12182.

(23) Liu, Y.; Ren, W.-M.; He, K.-K.; Lu, X.-B. Crystalline $\mathrm{CO}_{2}$-based Polycarbonates prepared from Racemic Catalyst Through Intramolecularly Interlocked Assembly. Nat. Commun. $\mathbf{2 0 1 5}, 5,5687$.

(24) Reports on MALDI-TOF analyses of terpolymers remain surprisingly limited. For an example, refer to: (a) Milani, B.; Scarel, A.; Durand, J.; Mestroni, G. MALDI-TOF Mass Spectrometry in the Study of CO/Aromatic Olefins Terpolymers. Macromolecules 2003, 36, 6295-6297. (b) Durand, J.; Scarel, A.; Milani, B.; Seraglia, R.; Gladiali, S.; Carfagna, C.; Binotti, B. Palladium-Promoted Carbon Monoxide/Ethylene/Styrene Terpolymerization Reaction: Throwing Light on the Different Reactivity of the Two Alkenes. Helv. Chim. Acta 2006, $89,1752-1771$.

(25) (a) Jutz, F.; Buchard, A.; Kember, M. R.; Fredriksen, S. B.; Williams, C. K. Mechanistic Investigation and Reaction Kinetics of the Low-pressure Copolymerization of Cyclohexene Oxide and Carbon Dioxide catalyzed by a Dizinc Complex. J. Am. Chem. Soc. 2011, 133, 17395-17405. (b) Decortes, A.; Haak, R. M.; Martín, C.; Martínez Belmonte, M.; Martin, E.; Benet-Buchholz, J.; Kleij, A. W. Copolymerization of $\mathrm{CO}_{2}$ and Cyclohexene Oxide Mediated by Yb(salen)-Based Complexes. Macromolecules 2015, 48, 8197-8207.

(26) NMR assignments (in ${ }^{1} \mathrm{H}$ and ${ }^{13} \mathrm{C} \mathrm{NMR}$ ) were carried out by using $2 \mathrm{D}$ experiments (COSY and HSQC) and the results compared with similar compounds in the literature, see for 
instance: Claudino, M.; Mathevet, J.-M.; Jonsson, M.; Johansson, M. Bringing D-Limonene to the Scene of Bio-based Thermoset Coatings via Free-radical Thiol-ene Chemistry: Macromonomer Synthesis, UV-curing and Thermo-mechanical Characterization. Polym. Chem. 2014, 5, 3245-3260.

(27) Note that these cross-linking reactions are fully reproducible following this approach; more details are provided in the Supporting Information: Table S4.

(28) The soluble cross-linked polymer CLP-10 was analyzed by GPC to assess the influence of the cross-linking process compared to the parent terpolymer T-10 $\left(M_{\mathrm{n}}=8.22 \mathrm{~kg} / \mathrm{mol}, D=1.24\right)$. After cross-linking both the $M_{\mathrm{n}}(14.6 \mathrm{~kg} / \mathrm{mol})$ and polydispersity $(\nexists=2.94)$ significantly increased.

(29) (a) Kulkarni, M.; Potrekar, R.; Kulkarni, R. A.; Vernekar, S. P. J. Synthesis and Characterization of Novel Polybenzimidazoles bearing Pendant Phenoxyamine Groups. Polym. Sci., Part A: Polym. Chem. 2008, 46, 5776-5793. (b) Kim, S.-K., Choi, S.-W.; Jeon, W. S.; Park, J. O.; Ko, T.; Chang, H.; Lee, J.-C. Cross-Linked Benzoxazine-Benzimidazole Copolymer Electrolyte Membranes for Fuel Cells at Elevated Temperature. Macromolecules, 2012, 45, $1438-1446$.

(30) Fiorani, G.; Stuck, M.; Martín, C.; Martin, E.; Martínez-Belmonte, M.; Escudero-Adán, E. C.; Kleij, A. W. Catalytic Coupling of Carbon Dioxide with Terpene Scaffolds: Access to Challenging Bio-Based Organic Carbonates. ChemSusChem 2016, 9, 1304-1311. 